\title{
Applied Systems Theory: Mathematical and numerical simulation of strength of thick-wall pipe by using static elastic problems
}

\author{
Natela Zirakashvili, \\ I. Vekua Institute of Applied Mathematics of Iv. Javakhishvili Tbilisi State University, \\ 2 University St., 0186 Tbilisi, 995 \\ Georgia
}

Received: April 1, 2021. Revised: August 17, 2021. Accepted: September 4, 2021. Published: September 6, 2021.

\begin{abstract}
In Systems Theory, the Mathematical and numerical simulation of strength of thick-wall pipe by using static elastic problems is an important problem and has attracted the attention of many researches, academicians and practitioners. the The present work studies the change in the strength of a quite long isotropic thick-wall pipe (circular cylinder) for the varying pipe diameter, wall thickness and material. The pipe is in the plane deformed state, i.e. plane deformation is considered. Based on the problems of statics of the theory of elasticity, a mathematical model to calculate the strength of the thick-wall pipe was developed and the problems of statics of the theory of elasticity were set and solved analytically in the polar coordinate system. The analytical solution was obtained by the method of separation of variables, which is presented by two harmonious functions. The dependence of the pipe strength on the thickness and material of the pipe wall, when (a) normal stress is applied to the internal boundary (internal pressure) and external boundary is free from stresses and (b) normal stress is applied to the external boundary (external pressure) and the internal boundary is free from stresses, is studied. In particular, the minimum thicknesses of the walls of homogeneous isotropic circular cylinders of different materials and diameters with a plane deformed mode when the pressures in the cylinders do not exceed the admissible values were identified. Some numerical results are presented as tables, graphs and relevant consideration.
\end{abstract}

Keywords - Applied Systems Theory, homogeneous isotropic thick-wall pipe, modeling of strength of thickwall pipe, separation variables method, static elastic problems.

\section{INTRODUCTION}

In Systems Theory, the Mathematical and numerical simulation of strength of thick-wall pipe by using static elastic problems is an important problem and has attracted the attention of many researches, academicians and practitioners. As it is known, all solid bodies have some or other property of strength and rigidity, i.e. the property supporting the body to maintain its unity and sizes under the action of external forces acting on it within certain limits. The study of the strength and stability of a thick-walled pipe under the impact of external forces may be considered as a classical problem of mechanics. The methods of the strength of materials are used to do practical calculations and identify the necessary, as they call, reliable sizes of the parts of the machines and various building structures. The methods of the theory of elasticity to calculate the strength and stability of some or other structure are also used commonly. Despite this, it is the study of the static problems of the theory of elasticity allowing obtaining a comprehensive mathematical model to calculate the strength of a thick-walled pipe (circular cylinder).

Thick-walled pipes are often used in many different branches of industry and they are often under internal pressure or external load. By varying the pipe parameters, the optimal values of parameters (diameter, wall thickness and elastic properties), due to which the pipe does not disintegrate (is not cracked), will be selected.

Many researchers have considered different problems for a thick-walled cylinder [1-11]. In paper [1], a simplified closedform solution has been obtained for hollow cylinders subjected to loads symmetrically distributed about an axis of the cross- 
section, acting in the radial direction over part of the circumference. Article [2] studies the behavior of rotating thick-walled cylinders made of rubber-like materials. In work [3], the authors consider the influence of the parameters of geometrical shape and properties of the materials on the limit load of a thick-walled cylinder. High internal pressures in a thick-walled cylinder produce great stresses on the internal surface of the cylinder. Therefore, the analysis of a thickwalled cylinder under the impact of high internal pressure, in particular, the identification of the stress concentration coefficient in a cylinder with and without pressure, is topical [4]. Work [5] studies the influence of pressure and deformation on creep stresses in a thick-walled cylinder, which is made up of functionally graded material and is subjected to twisting. The problems of thick-walled round cylinders under internal pressure has been discussed by many authors [6-11] for the theory of isotropic plasticity and creep.

The present paper studies the dependence of the strength of quite a long thick-walled circular cylinder on the wall thickness and material (elastic properties). In particular, the minimum thicknesses of the walls of the cylinders made up of various homogeneous isotropic materials (for example: steel, copper, aluminum and grey cast iron) and of different diameters $(3 \mathrm{~cm}, 5 \mathrm{~cm}$ and $10 \mathrm{~cm})$ in the state of plane deformation, with which the stresses in the cylinders do not exceed the admissible values, is identified.

In order to solve the boundary value and boundary-contact problems for the regions with a curvilinear boundary, it is feasible to consider these problems in a appropriate curvilinear coordinate system. For instance, the problems for the regions bounded with a circle or its parts are considered in a polar coordinate system [2-15] for the areas bounded with an ellipse, hyperbola or their parts, the problems are considered in an elliptic coordinate system [16-25]; for the areas bounded with a parabola or its parts, they are considered in a parabolic coordinate system [26,27], while for the areas bounded with eccentric circles, the problems are considered in a bipolar coordinate system [28-30]. Thus, the boundary value problems for a circular cylinder in the state of plane deformation are considered in a polar coordinate system.

The second section of the present article gives a system of equilibrium equations of elastic plane deformation state in a circular cylindrical coordinate system, Hook's Law and posing the problems.

In the third section, by using the method of separation of variables, an analytical solution of one of the posed problems is obtained, which is presented as two harmonic functions.

The fourth section gives the numerical simulation and graphs with relevant explanations. The numerical results and appropriate graphics are obtained by using MATLAB software

And finally, the fifth section (the Conclusion) gives the main results given in the present work and prospects of their use in practice.

\section{EQUATIONS OF ELASTIC PLANE DEFORMED STATE IN CIRCULAR CYLINDRICAL COORDINATE SYSTEM AND POSING BOUNDARY-VALUE PROBLEMS}

In case of absence of volume forces, the equilibrium equations of elastic plane deformed state are given as follows in a circular cylindrical coordinate system [31]:

$$
\begin{aligned}
& R_{r, r}+\frac{1}{r} R_{\alpha, \alpha}+\frac{R_{r}}{r}-\frac{A_{\alpha}}{r}=0, \\
& \frac{1}{r} A_{\alpha, \alpha}+R_{\alpha, r}+\frac{2 R_{\alpha}}{r}=0,
\end{aligned}
$$

where $R_{r}, A_{\alpha}, Z_{z}$ are normal stresses and $R_{\alpha}=A_{r}, A_{z}=Z_{\alpha}, R_{z}=Z_{r}$ are tangential stresses.

When writing down equations (1), it is considered that the stresses and deformations in the elastic area $\widetilde{\Omega}=\left\{r_{1}<r<r_{2}, 0 \leq \alpha<2 \pi, 0<z<z_{1}\right\}$ under consideration do not change along coordinate $z$. In this case, when $z=0$ and $z=z_{1}$, the following conditions must be true: $w=0, Z_{r}=0, Z_{\alpha}=0$, where $w$ is the component of vector $\vec{U}$ along coordinate $z$ ( $z_{1}$ is any positive number).

Thus, we have a plane deformed state when there remain only radial $u(r, \alpha)$ and circular $\mathrm{v}(r, \alpha)$ components of three components of vector $\overrightarrow{\mathrm{U}}$ and normal stresses $R_{r}, A_{\alpha}$ and tangential stress $R_{\alpha}$ of the stress tensor components.

The stresses are expressed with the following equations by means of displacements [32]:

$$
\begin{aligned}
& R_{r}=\lambda\left(u_{, r}+\frac{u}{r}+\frac{1}{r} \mathrm{v}_{, \alpha}\right)+2 \mu u_{, r}, \\
& A_{\alpha}=\lambda\left(u_{, r}+\frac{u}{r}+\frac{1}{r} \mathrm{v}_{, \alpha}\right)+2 \mu\left(\frac{1}{\mathrm{r}} \mathrm{v}_{, \alpha}+\frac{u}{r}\right), \\
& R_{\alpha}=\mu\left(\mathrm{v}_{, r}-\frac{\mathrm{v}}{r}+\frac{1}{r} u_{, \alpha}\right) .
\end{aligned}
$$

Here $\lambda=\frac{v E}{(1+v)(1-2 v)}, \quad \mu=\frac{E}{2(1+v)}$, where $E$ is modulus of elasticity and $v$ is Poisson's ratio.

By virtue of the symmetry principle, finally the problem will be posed for quarter of circular ring $\widetilde{\Omega}$ : $\Omega=\left\{r_{1}<r<r_{2}, 0<\alpha<\frac{\pi}{2}\right\}$. Due to the plane deformation, there is no third dimension in $\Omega$ area.

Let us write down the boundary conditions for the quarter of a circular ring:

$$
\alpha=0, \alpha=\frac{\pi}{2}: \mathrm{v}=0, A_{r}=0 \Leftrightarrow \mathrm{v}=0, u_{, \alpha}=0
$$




$$
\begin{aligned}
r=r_{k}, \quad k=1,2: \quad \text { a) } R_{r}=F_{11}^{(k)}(\alpha), \quad R_{\alpha}=F_{12}^{(k)}(\alpha) \text { or } \\
\text { b) } u=F_{21}^{(k)}(\alpha), \quad v=F_{22}^{(k)}(\alpha), \text { or } \\
\text { c) } v=F_{22}^{(k)}(\alpha), \quad R_{r}=F_{11}^{(k)}(\alpha) \text {, or } \\
\text { d) } u=F_{21}^{(k)}(\alpha), \quad R_{\alpha}=F_{12}^{(k)}(\alpha) .
\end{aligned}
$$

It is implied that $F_{11}^{(k)}(\alpha), F_{12}^{(k)}(\alpha)$ functions, together with their first order derivatives, and $F_{21}^{(k)}(\alpha), F_{22}^{(k)}(\alpha)$, functions, together with their first and second order derivatives, decompose into absolute and uniformly convergent Fourier trigonometric series.

Let us consider one of the posed problems, e.g. problem (1), (3), (4a). Other problems can be solved similarly.

\section{SOlVING PROBLem (1), (3), (4 a )}

Let us consider a plane deformed homogeneous isotropic cylinder with $r_{1}$ internal radius and $r_{2}$ external radius. For generality, let us assume that the cylinder is loaded simultaneously with internal $p_{1}(\alpha)$ and external $p_{2}(\alpha)$ stresses. Let us imply that the cylinder is quite long for stress $Z_{z}$ to be distributed equally across the cross section and the influence of the cylinder bed on the radial displacement is very small. Besides, we consider the case when $Z_{z}=0$. Mathematically, this problem is described with formulae (1), (2), (3), (4a). So, we must find the solution of the system of equilibrium equations (1) in $\Omega=\left\{r_{1}<r<r_{2}, 0<\alpha<\frac{\pi}{2}\right\}$ area, which satisfies (3), (4a) boundary conditions. We can give the numerical realization of the given problem based on the solutions obtained by using two methods (analytical solution obtained with a method of separation of variables and Lamé solution).

\section{A. Obtaining Analytical Solutions by Using the Method of Separation of Variables}

In case of absence of volume forces, a system of equilibrium equations written in polar coordinates is obtained as a result of projecting a known differential equation of the equilibrium of a homogeneous elastic isotropic body $(\lambda+2 \mu) \operatorname{grad} \operatorname{div} \vec{U}-\mu \operatorname{rot} \operatorname{rot} \vec{U}=0$ on the coordinate axes of polar $r, \alpha$ coordinate system. The given system of equilibrium equations can be written down by using functions $K, B, u, \mathrm{v}$ as follows:
(a) $r K_{, r}-B_{, \alpha}=0$
(c) $u_{, r}+\frac{u}{r}+\frac{1}{r} \mathrm{v}_{, \alpha}=\frac{K}{\lambda+2 \mu}$
(b) $r B_{, r}+K_{, \alpha}=0$,
(d) $\mathrm{v}_{, r}+\frac{\mathrm{v}}{r}-\frac{1}{r} u_{, \alpha}=\frac{B}{\mu}$,

where

$$
B=B_{z}=\operatorname{rot}_{z} \vec{U}=\mathrm{v}_{, r}-\frac{1}{r} u_{, \alpha}+\frac{\mathrm{v}}{r}, K=\operatorname{div} \vec{U}=u_{, r}+\frac{u}{r}+\frac{1}{r} \mathrm{v}_{, \alpha} .
$$

Theorem 1 In the class of regular functions, the general solution of system (5) for the considered class of boundary value problems of the theory of elasticity is presented by means of two $\varphi_{1}$ and $\varphi_{2}$ harmonic functions as follows:

$$
\begin{aligned}
& u=r^{3} \varphi_{1, r r}+\frac{\lambda-\mu}{\lambda+\mu} r^{2} \varphi_{1, r}+\varphi_{2, r}, \\
& \mathrm{v}=r^{2} \varphi_{1, r \alpha}+\frac{2(\lambda+2 \mu)}{\lambda+\mu} r \varphi_{1, \alpha}+\frac{1}{r} \varphi_{2, \alpha},
\end{aligned}
$$

where (see Appendix $C$ )

$$
\begin{aligned}
\varphi_{i}= & \sum_{\mathrm{k}=1}^{\infty}\left[\left(A_{i k} r^{k}+B_{i k} r^{-k}\right)_{\cos (k \alpha)}^{\sin (k \alpha)}+\left(\widetilde{A}_{i k} e^{\alpha k}+\widetilde{B}_{i k} e^{-k \alpha}\right)_{\cos (k \ln r)}^{\sin (k \ln r)}\right] \text { (7) } \\
& +a_{i 0} \alpha \ln r+b_{i 0} \ln r+c_{i 0} \alpha+d_{i 0}, \quad i=1,2 .
\end{aligned}
$$

Proof Let us satisfy equation (5b) identically:

$$
K=r\left(r \varphi_{1}\right)_{, r}, B=-\left(r \varphi_{1}\right)_{, r \alpha},
$$

where $\varphi_{1}$ is a harmonic function. It is easy to show that $\left(r \varphi_{1}\right)_{, r}$ is harmonic (Appendix A), i.e. $\Delta(r \varphi)_{, r}=0$.

Let us insert (8) in (5c) and (5d). We will obtain:

$$
\begin{aligned}
& u_{, r}+\frac{u}{r}+\frac{1}{r} \mathrm{v}_{, \alpha}=\frac{1}{\lambda+2 \mu} r\left(r \varphi_{1}\right)_{, r r}, \\
& (r \mathrm{~V})_{, r}-\left(u-\frac{r}{\mu}\left(r \varphi_{1}\right)_{, r}\right)_{, \alpha}=0
\end{aligned}
$$

Let us satisfy second equation of (9) identically:

$$
\mathrm{v}=\frac{1}{r} \widetilde{\varphi}_{2, \alpha}, \quad u=\widetilde{\varphi}_{2, r}+\frac{r}{\mu}\left(r \varphi_{1}\right)_{, r} .
$$

Let us insert (10) in the first equation of (9). Following simple transformations, we will obtain:

$$
\Delta \widetilde{\varphi}_{2}=-\frac{\lambda+\mu}{\mu(\lambda+2 \mu)} r\left(r \varphi_{1}\right)_{, r r}-\frac{2}{\mu}\left(r \varphi_{1}\right)_{, r} .
$$

By considering the proved formulas in Appendix B, we will obtain:

$$
\begin{aligned}
& \Delta \widetilde{\varphi}_{2}=-\frac{\lambda+\mu}{\mu(\lambda+2 \mu)}\left(r^{2} \varphi_{1, r}\right)_{, r}-\frac{2}{\mu}\left(r \varphi_{1}\right)_{, r} ; \\
& \Delta\left(\widetilde{\varphi}_{2}+\frac{\lambda+\mu}{4 \mu(\lambda+2 \mu)} r^{3} \varphi_{1, r}+\frac{r^{2}}{2 \mu} \varphi_{1}\right)=0 ; \\
& \widetilde{\varphi}_{2}=\varphi_{2}-\frac{\lambda+\mu}{4 \mu(\lambda+2 \mu)} r^{3} \varphi_{1, r}-\frac{r^{2}}{2 \mu} \varphi_{1},
\end{aligned}
$$

where $\Delta \varphi_{2}=0$.

Let $\quad$ us take $\quad \varphi_{1}=-\frac{4 \mu(\lambda+2 \mu)}{\lambda+\mu} \varphi_{1}$, then $\widetilde{\varphi}_{2}=\varphi_{2}+r^{3} \varphi_{1, r}+\frac{2(\lambda+2 \mu)}{\lambda+\mu} r^{2} \varphi_{1}$, where $\frac{2(\lambda+2 \mu)}{\lambda+\mu}=4(1-v)$.

Following some elementary transformations, we will finally obtain (6), i.e. theorem 1 is proved.

After inserting (6) in (2) and doing simple transformations, the stress tensor components will be written down as follows: 


$$
\begin{aligned}
R_{r}= & (\lambda+2 \mu) r^{3} \varphi_{1, r r r}+\lambda r \varphi_{1, r \alpha \alpha} \\
& +\frac{5 \lambda^{2}+11 \lambda \mu+4 \mu^{2}}{\lambda+\mu} r^{2} \varphi_{1, r r}+\lambda \frac{2(\lambda+2 \mu)}{\lambda+\mu} \varphi_{1, \alpha \alpha} \\
& +\frac{3 \lambda^{2}+\lambda \mu-4 \mu^{2}}{\lambda+\mu} r \varphi_{1, r}+2 \mu \varphi_{2, r r}, \\
A_{\alpha}= & \lambda r^{3} \varphi_{1, r r r}+(\lambda+2 \mu) r \varphi_{1, r \alpha \alpha} \\
& +\frac{5 \lambda^{2}+5 \lambda \mu+2 \mu^{2}}{\lambda+\mu} r^{2} \varphi_{1, r r}+\frac{2(\lambda+2 \mu)^{2}}{\lambda+\mu} \varphi_{1, \alpha \alpha} \\
& +\frac{(\lambda-\mu)(3 \lambda+2 \mu)}{\lambda+\mu} r \varphi_{1, r}+\frac{2 \mu}{r^{2}} \varphi_{2, \alpha \alpha}+\frac{2 \mu}{r} \varphi_{2, r}, \\
R_{\alpha}= & 2 \mu r^{2} \varphi_{1, r r \alpha}+4 \mu r \varphi_{1, r \alpha}+\frac{2 \mu}{r} \varphi_{2, r \alpha}-\frac{2 \mu}{r^{2}} \varphi_{2, \alpha} .
\end{aligned}
$$

By considering condition (3), expression (7) will be formulated as follows:

$$
\varphi_{i}=\ln r b_{i 0}+\sum_{\mathrm{k}=1}^{\infty}\left[\left(A_{i k}\left(\frac{r}{r_{2}}\right)^{n}+B_{i k}\left(\frac{r_{1}}{r}\right)^{n}\right) \cos (n \alpha)\right], i=1,2, n=2 k .
$$

After inserting (12) in (6) and (11), the following expressions for displacements will be obtained:

$$
\begin{aligned}
u= & \frac{-2 \mu}{\lambda+\mu} r b_{10}+\frac{1}{r} b_{20} \\
& +\sum_{k=1}^{\infty} n\left[r\left(n-\frac{2 \mu}{\lambda+\mu}\right)\left(\frac{r}{r_{2}}\right)^{n} A_{1 k}+r\left(n+\frac{2 \mu}{\lambda+\mu}\right)\left(\frac{r_{1}}{r}\right)^{n} B_{1 k}\right. \\
& \left.+\frac{1}{r}\left(\frac{r}{r_{2}}\right)^{n} A_{2 k}-\frac{1}{r}\left(\frac{r_{1}}{r}\right)^{n} B_{2 k}\right] \cos (n \alpha), \\
\mathrm{v}= & \sum_{k=1}^{\infty} n\left[-r\left(n+\frac{\lambda+2 \mu}{\lambda+\mu}\right)\left(\frac{r}{r_{2}}\right)^{n} A_{1 k}+r\left(n-\frac{\pi+2 \mu}{\lambda+\mu}\right)\left(\frac{r_{1}}{r}\right)^{n} B_{1 k}\right. \\
& \left.-\frac{1}{r}\left(\frac{r}{r_{2}}\right)^{n} A_{2 k}-\frac{1}{r}\left(\frac{r_{1}}{r}\right)^{n} B_{2 k}\right] \sin (n \alpha), n=2 k .
\end{aligned}
$$

And the following expressions will be obtained for stresses:

$$
\begin{aligned}
R_{r}= & -4 \mu b_{10}-\frac{2 \mu}{r^{2}} b_{20} \\
& +\sum_{k=1}^{\infty}\left\{2 \mu n[n(n-1)-2]\left(\frac{r}{r_{2}}\right)^{n} A_{1 k}+2 \mu n[n(n+1)-2]\left(\frac{r_{1}}{r}\right)^{n} B_{1 k}\right. \\
& \left.+2 \mu n(n-1) \frac{1}{r^{2}}\left(\frac{r}{r_{2}}\right)^{n} A_{2 k}+2 \mu n(n+1) \frac{1}{r^{2}}\left(\frac{r_{1}}{r}\right)^{n} B_{2 k}\right\} \cos (n \alpha), \\
A_{\alpha}= & -4 \mu b_{10}+\frac{2 \mu}{r^{2}} b_{20} \\
& +\sum_{k=1}^{\infty}\left\{-2 n\left[\mu n(n+3)+\frac{\lambda^{2}+2 \mu^{2}+\lambda \mu}{\lambda+\mu}\right]\left(\frac{r}{r_{2}}\right)^{n} A_{1 k}\right. \\
& +2 n\left[\mu n(n-3)+\frac{\lambda^{2}+2 \mu^{2}+\lambda \mu}{\lambda+\mu}\right]\left(\frac{r_{1}}{r}\right)^{n} B_{1 k} \\
& \left.-2 \mu n(n-1) \frac{1}{r^{2}}\left(\frac{r}{r_{2}}\right)^{n} A_{2 k}-2 \mu n(n+1) \frac{1}{r^{2}}\left(\frac{r_{1}}{r}\right)^{n} B_{2 k}\right\} \cos (n \alpha),
\end{aligned}
$$

$$
\begin{aligned}
R_{\alpha}= & \sum_{k=1}^{\infty}-2 \mu n\left\lfloor n(n+1)\left(\frac{r}{r_{2}}\right)^{n} A_{1 k}+n(n-1)\left(\frac{r_{1}}{r}\right)^{n} B_{1 k}\right. \\
& \left.+(n-1) \frac{1}{r^{2}}\left(\frac{r}{r_{2}}\right)^{n} A_{2 k}-(n+1) \frac{1}{r^{2}}\left(\frac{r_{1}}{r}\right)^{n} B_{2 k}\right] \sin (n \alpha), \quad n=2 k .
\end{aligned}
$$

From boundary conditions (4a), an infinite system of linear algebraic equations to unknown coefficients $b_{10}, b_{20}$, $A_{i k}, B_{i k}(i=1,2, k=1,2, \ldots)$ will be obtained.

$$
\begin{aligned}
& -4 \mu b_{10}-\frac{2 \mu}{r_{1}^{2}} b_{20} \\
& +\sum_{k=1}^{\infty}\left[L_{1}\left(\frac{r_{1}}{r_{2}}\right)^{n} A_{1 k}+L_{2} B_{1 k}+L_{3} \frac{1}{r_{1}^{2}}\left(\frac{r_{1}}{r_{2}}\right)^{n} A_{2 k}+L_{4} \frac{1}{r_{1}^{2}} B_{2 k}\right] \\
& \cdot \cos (n \alpha)=F_{11}^{(1)}(\alpha)
\end{aligned}
$$

$$
\begin{gathered}
\sum_{k=1}^{\infty}\left\lfloor-n L_{4}\left(\frac{r_{1}}{r_{2}}\right)^{n} A_{1 k}-n L_{3} B_{1 k}-L_{3} \frac{1}{r_{1}^{2}}\left(\frac{r_{1}}{r_{2}}\right)^{n} A_{2 k}+L_{4} \frac{1}{r_{1}^{2}} B_{2 k}\right] \\
\cdot \sin (n \alpha)=F_{12}^{(1)}(\alpha) . \\
-4 \mu b_{10}-\frac{2 \mu}{r_{2}^{2}} b_{20} \quad \cdot \cos (n \alpha)=F_{11}^{(2)}(\alpha), \\
+\sum_{k=1}^{\infty}\left[L_{1} A_{1 k}+L_{2}\left(\frac{r_{1}}{r_{2}}\right)^{n} B_{1 k}+L_{3} \frac{1}{r_{2}^{2}} A_{2 k}+L_{4} \frac{1}{r_{2}^{2}}\left(\frac{r_{1}}{r_{2}}\right)^{n} B_{2 k}\right] \\
\sum_{k=1}^{\infty}\left\lfloor-n L_{4} A_{1 k}-n L_{3}\left(\frac{r_{1}}{r_{2}}\right)^{n} B_{1 k}-L_{3} \frac{1}{r_{2}^{2}} A_{2 k}+L_{4} \frac{1}{r_{2}^{2}}\left(\frac{r_{1}}{r_{2}}\right)^{n} B_{2 k}\right\rfloor \\
\cdot \sin (n \alpha)=F_{12}^{(2)}(\alpha) .
\end{gathered}
$$

Here:

$L_{1}:=2 \mu n[n(n-1)-2], \quad L_{2}:=2 \mu n[n(n+1)-2], L_{3}:=2 \mu n(n-1)$, $L_{4}:=2 \mu n(n+1), n=2 k$.

After decomposing the functions on the right side of equations (15) into Fourier trigonometric series, as a result of equating the expressions at the same trigonometric functions in both sides of the equations, an infinite system of linear algebraic equations will be obtained. The principal matrix of such a system is a block-diagonal one (See Fig. 1). Block $D_{1}$ is $2 \times 2$ - and blocks $D_{i}, i=2,3, \ldots$ are $4 \times 4$-dimensional matrixes and the relevant determinants differ from zero. 


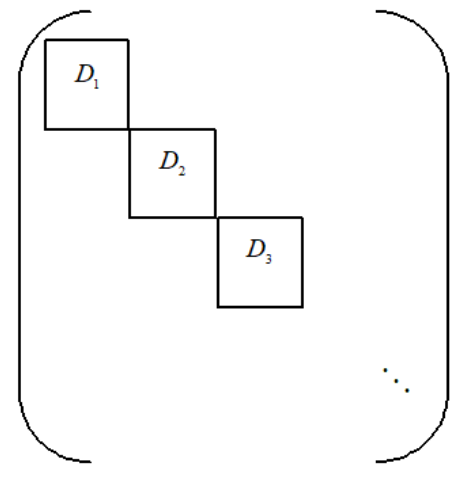

Fig. 1. Plan of the principal matrix of the infinite system of equations (15)

\section{B. Lamé problem solution}

For generality, let us assume that a thick-walled cylinder is loaded with internal $p_{1}=$ constant and external $p_{2}=$ constant forces at the same time. In this case, both, the deformations and stress distribution will be symmetrical, as any radial section is a plane of symmetry and therefore, there are no tangential stresses on these sections, i.e. $A_{r}=R_{\alpha}=0$. The problem to determine displacements and stresses in a thick-walled cylinder is known as Lamé problem, which gained the solution of this problem [33-35].

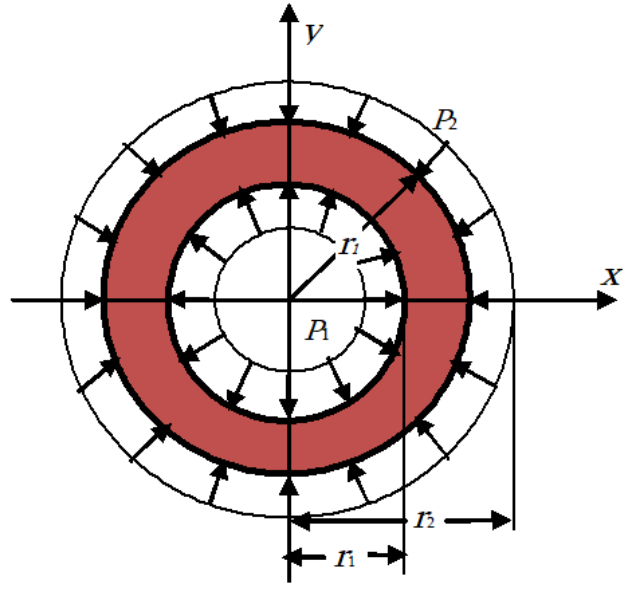

Fig. 2. Plan of load on the cross section of a plane deformed thick-walled circular cylinder

$$
\begin{aligned}
& u=\frac{1-\mu}{E} \frac{p_{1} r_{1}^{2}-p_{2} r_{2}^{2}}{r_{2}^{2}-r_{1}^{2}} r+\frac{1+\mu}{E} \frac{r_{1}^{2} r_{2}^{2}}{r} \frac{p_{1}-p_{2}}{r_{2}^{2}-r_{1}^{2}} \\
& R_{r}=\frac{p_{1} r_{1}^{2}-p_{2} r_{2}^{2}}{r_{2}^{2}-r_{1}^{2}}-\frac{r_{1}^{2} r_{2}^{2}}{r^{2}} \frac{p_{1}-p_{2}}{r_{2}^{2}-r_{1}^{2}} \\
& A_{\alpha}=\frac{p_{1} r_{1}^{2}-p_{2} r_{2}^{2}}{r_{2}^{2}-r_{1}^{2}}+\frac{r_{1}^{2} r_{2}^{2}}{r^{2}} \frac{p_{1}-p_{2}}{r_{2}^{2}-r_{1}^{2}}
\end{aligned}
$$

\section{NUMERICAL SIMULATION AND CONSIDERATION OF RESULTS}

The present section gives the results of the calculation of a thick-walled circular homogeneous isotropic body under the impact of internal pressure or external forces on strength. The strength calculation of a cylinder implies determining the minimum thicknesses of the walls of the thick-walled pipes of different materials and different diameters when the stresses in the body do not exceed the admissible value [34,35], i.e. when the pipe will not disintegrate (is not cracked). So, the numerical results of the boundary-value problems given here and relevant graphs for the quarter circular ring when (1) constant normal load is given on the internal boundary and the external boundary is free from loads, (2) constant normal load is given on the external boundary and internal boundary is free from loads. Thus, the dependence of the strength of a plane deformed thick-walled circular cylinder on the thickness and material of the wall (elastic properties) is studied. Its relevant numerical solutions are obtained based on (a) the analytical solution obtained with the method of separation of variables (See formulas (13) and (14)) and (b) Lamé solution (See formulas (16), (17)).

Note. As Lamé solutions are obtained for the cylinders under constant normal load, in order to compare the numerical results obtained by using analytical and numerical methods with the numerical results based on Lamé solutions, the numerical and visual realization is done for the cylinder under constant normal load.

As it is known, a cylinder is called a thick-walled one, for which the ratio of its wall thickness with its internal diameter is no less that $1 / 20$. Therefore, we must take $0<r_{1} \leq \frac{10}{11} r_{2}$. We will have the variation of the wall thickness, if we fix $r_{2}$ and confer $r_{1}$ values from range $0<r_{1} \leq \frac{10}{11} r_{2}$. The numerical values are obtained for the following data: $r_{2}=1.5 \mathrm{~cm}, 2.5 \mathrm{~cm}, 5 \mathrm{~cm}, \quad p=-100 \mathrm{~kg} / \mathrm{cm}^{2}, \quad$ and $E=2 \cdot 10^{6} \mathrm{~kg} / \mathrm{cm}^{2}, \quad v=0.3$ for steel, $E=1.1 \cdot 10^{6} \mathrm{~kg} / \mathrm{cm}^{2}$, $v=0.32$ for copper, $E=0.7 \cdot 10^{6} \mathrm{~kg} / \mathrm{cm}^{2}, \quad v=0.34$ for aluminum and $E=0.7 \cdot 10^{6} \mathrm{~kg} / \mathrm{cm}^{2}, v=0.25$ for grey cast iron.

\section{A. Strength calculation for a thick-walled cylinder under} the impact of internal pressure

Let us insert $\quad F_{11}^{(1)}(\alpha)=-p, \quad F_{12}^{(1)}(\alpha)=0, \quad F_{11}^{(2)}(\alpha)=0$, $F_{12}^{(2)}(\alpha)=0$ in formulas (15). We will obtain the following system of equations in relation to $b_{10}, b_{20}, A_{1 k}$ and $B_{1 k}$ :

$-4 \mu b_{10}-\frac{2 \mu}{r_{1}^{2}} b_{20}=-p$

$-n L_{4}\left(\frac{r_{1}}{r_{2}}\right)^{n} A_{1 k}-n L_{3} B_{1 k}-L_{3} \frac{1}{r_{1}^{2}}\left(\frac{r_{1}}{r_{2}}\right)^{n} A_{2 k}+L_{4} \frac{1}{r_{1}^{2}} B_{2 k}=0$, 


$$
\begin{aligned}
&-4 \mu b_{10}-\frac{2 \mu}{r_{2}^{2}} b_{20}=0, \\
&-n L_{4} A_{1 k}-n L_{3}\left(\frac{r_{1}}{r_{2}}\right)^{n} B_{1 k}-L_{3} \frac{1}{r_{2}^{2}} A_{2 k}+L_{4} \frac{1}{r_{21}^{2}}\left(\frac{r_{1}}{r_{2}}\right)^{n} B_{2 k}=0, \\
& k=1,2, \ldots, \quad n=2 k
\end{aligned}
$$

Here:

$$
\begin{aligned}
& L_{1}:=2 \mu n[n(n-1)-2], \quad L_{2}:=2 \mu n[n(n+1)-2], \\
& L_{3}:=2 \mu n(n-1), \quad L_{4}:=2 \mu n(n+1), \quad n=2 k .
\end{aligned}
$$

From here:

$$
\begin{aligned}
& b_{10}=-\frac{p r_{1}^{2}}{4 \mu\left(r_{2}^{2}-r_{1}^{2}\right)}, \quad b_{20}=\frac{p r_{1}^{2} r_{2}^{2}}{2 \mu\left(r_{2}^{2}-r_{1}^{2}\right)} . \\
& A_{i k}=0, \quad B_{i k}=0, \quad i=1,2, \quad k=1,2, \ldots .
\end{aligned}
$$

After inserting expression (18) in equations (13) and (14), the following expressions will be obtained:

$$
\begin{aligned}
& u=\frac{1}{2(\lambda+\mu)} r \frac{p r_{1}^{2}}{r_{2}^{2}-r_{1}^{2}}+\frac{1}{2 \mu} \frac{1}{r} \frac{p r_{1}^{2} r_{2}^{2}}{r_{2}^{2}-r_{1}^{2}} . \\
& R_{r}=\frac{p r_{1}^{2}}{r_{2}^{2}-r_{1}^{2}}-\frac{r_{1}^{2} r_{2}^{2}}{r^{2}} \frac{p}{r_{2}^{2}-r_{1}^{2}}, \\
& A_{\alpha}=\frac{p r_{1}^{2}}{r_{2}^{2}-r_{1}^{2}}+\frac{1}{r^{2}} \frac{p r_{1}^{2} r_{2}^{2}}{r_{2}^{2}-r_{1}^{2}}, \\
& R_{\alpha}=0 .
\end{aligned}
$$

Displacements and stresses at any point of the considered area are obtained from equations (19) and (20).

If in Lamé solutions, in (16), (17), we take $p_{1}=p, \quad p_{2}=0$, then expressions (16), (17) will be given as (19) and (20).

\begin{tabular}{|c|c|c|c|c|c|c|}
\hline \multirow{2}{*}{ Material } & \multicolumn{3}{|c|}{$\begin{array}{l}\text { Minimum admissible wall } \\
\text { thickness }\left(r_{2}-r_{1}\right)\end{array}$} & \multirow{2}{*}{$\begin{array}{c}E, \\
\mathrm{Kg} / \mathrm{cm}^{2}\end{array}$} & \multirow{2}{*}{$v$} & \multirow{2}{*}{$\begin{array}{l}\text { Admiss } \\
\text { ible } \\
\text { stress, } \\
\mathrm{Kg} / \mathrm{cm}^{2}\end{array}$} \\
\hline & $r_{2}=1,5 \mathrm{~cm}$ & $r_{2}=2,5 \mathrm{~cm}$ & $r_{2}=5 \mathrm{~cm}$ & & & \\
\hline Steel & 0.1813636 & 0.3022727 & 0.6545455 & $2 \cdot 10^{6}$ & 0.3 & 713.801 \\
\hline Copper & 0.2263636 & 0.3772727 & 0.7545455 & $1.1 \cdot 10^{6}$ & 0.32 & 611.830 \\
\hline Aluminum & 0.2713636 & 0.4522727 & 0.8545455 & $0.7 \cdot 10^{6}$ & 0.34 & 509.858 \\
\hline $\begin{array}{l}\text { Grey } \\
\text { cast } \\
\text { iron }\end{array}$ & 0.2713636 & 0.4522727 & 0.8545455 & $0.7 \cdot 10^{6}$ & 0.25 & 509.858 \\
\hline
\end{tabular}

By using (16), (17) formulas, or (19) and (20) formulas, we obtain the minimum wall thickness of a circular steel, copper, aluminum and grey cast iron rings (i.e. of a circular cylinder wall), at which the stresses produced in the body do not exceed admissible values, when $r_{2}=1,5 ; 2,5 ; 5 \mathrm{~cm}$. The obtained numerical values are presented in table 1 .

TABIE I. Minimum admissible thicknesses of a cylinder wall at which the stresses produced in the cylinders of different materials and diameters do not exceed admissible values (internal load)

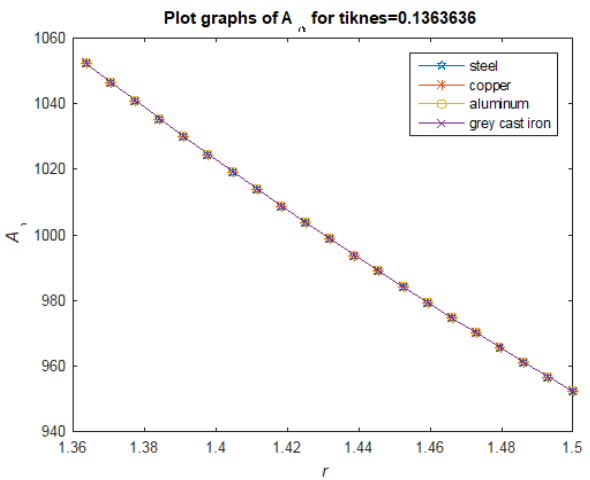

(a)

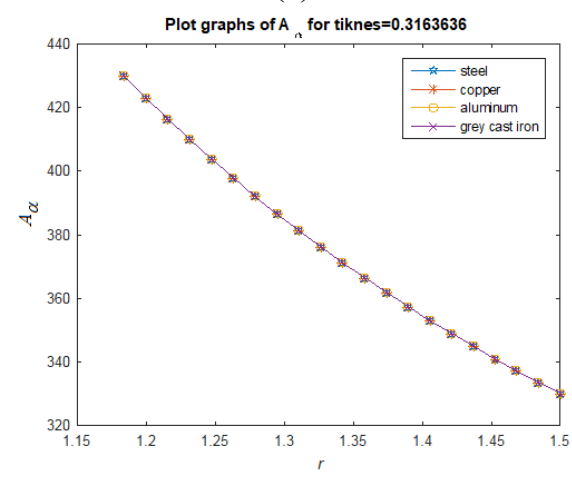

(b)

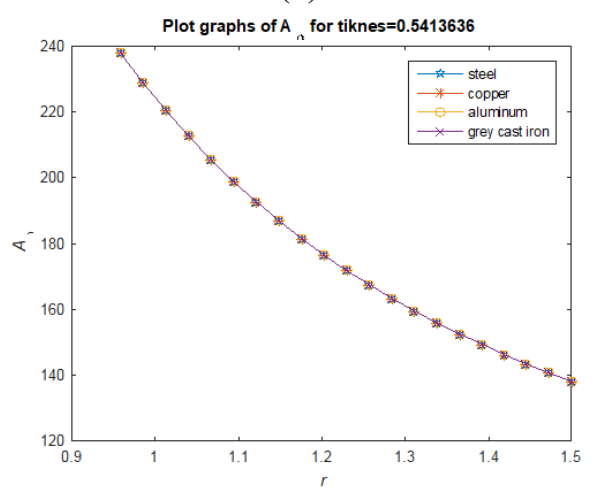

(c)

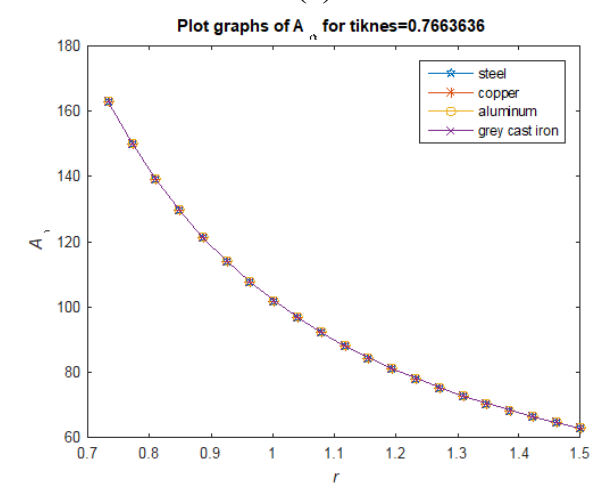

(d)

Fig. 3. Shearing stresses $A_{\alpha}$ along the radii of the walls of steel, copper, aluminum and grey iron circular pipes when the thickness of each wall is approximately: a) $0,136 \mathrm{~cm}, \mathrm{~b}) 0,316$ $\mathrm{cm}, \mathrm{c}) 0,541 \mathrm{~cm}$ and d) $0,766 \mathrm{~cm}\left(r_{2}=1,5\right.$ and internal pressure) 


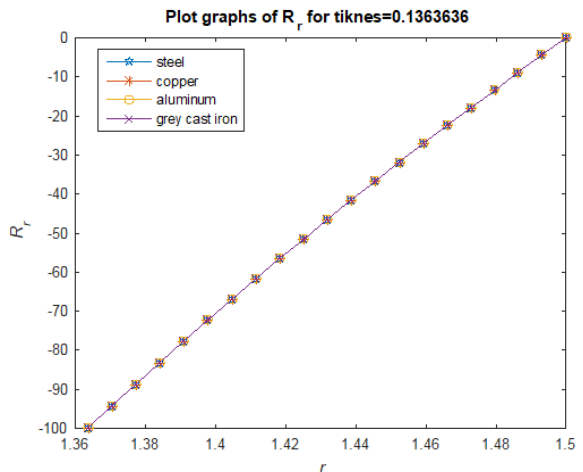

(a)

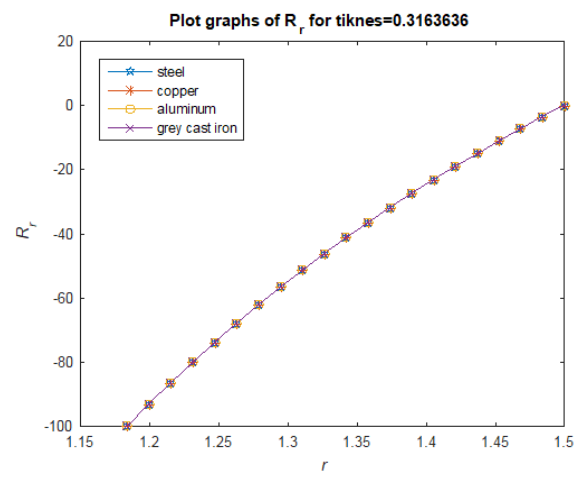

(b)

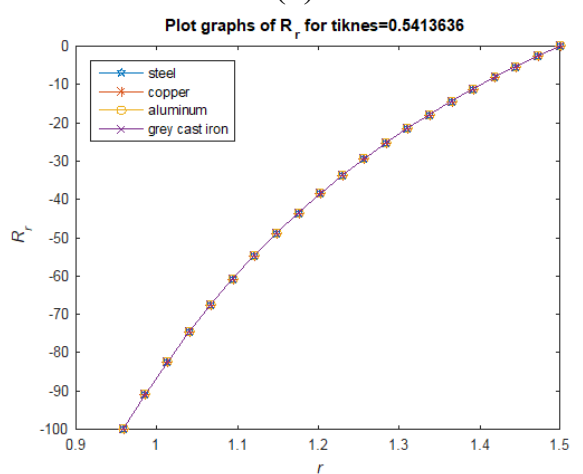

(c)

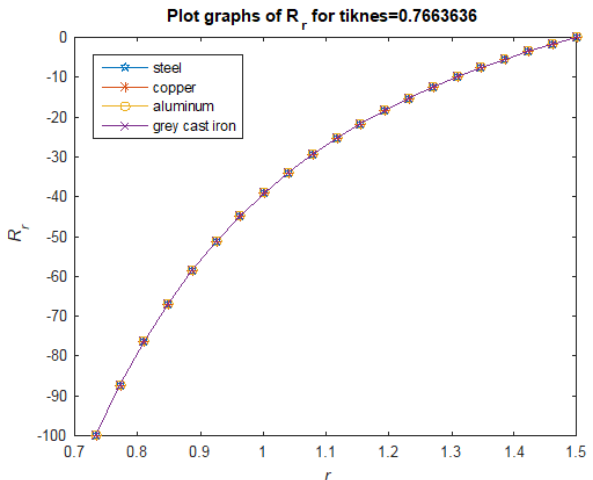

(d)

Fig. 4. Normal stresses $R_{r}$ along the radii of the walls of steel, copper, aluminum and grey iron circular pipes when the thickness of each wall is approximately: a) $0,136 \mathrm{~cm}, \mathrm{~b}) 0,316$ $\mathrm{cm}, \mathrm{c}) 0,541 \mathrm{~cm}$ and d) $0,766 \mathrm{~cm}\left(r_{2}=1,5\right.$ and internal pressure)

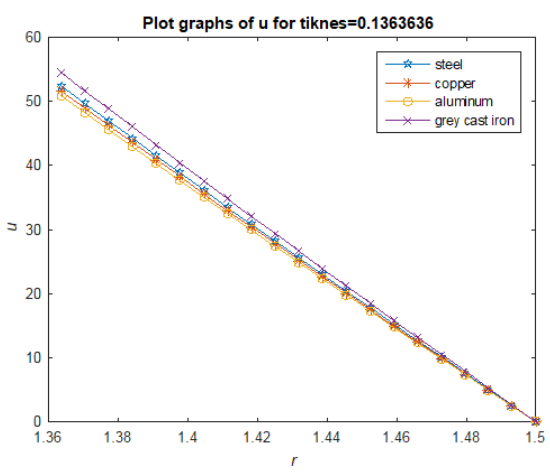

(a)

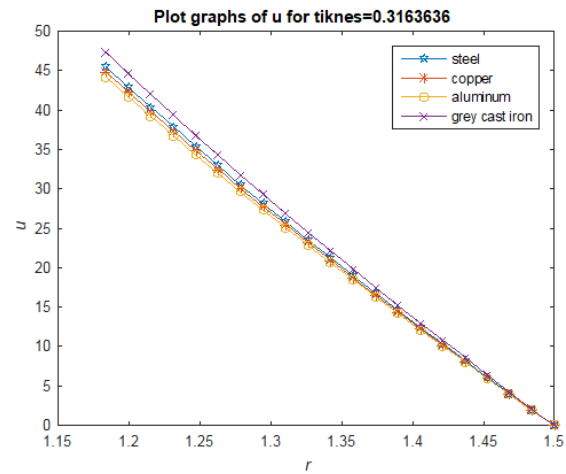

(b)

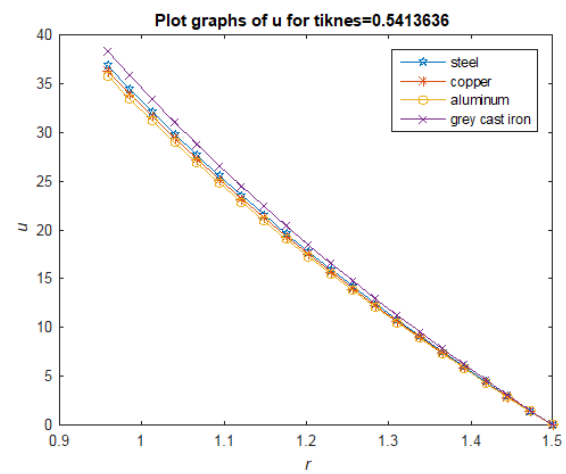

(c)

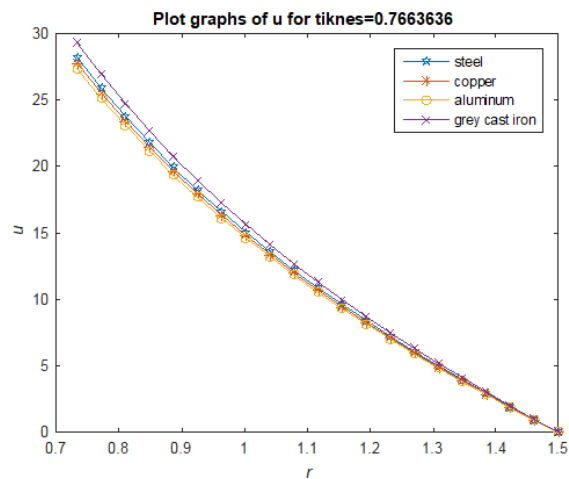

(d)

Fig. 5. Normal displacements $u$ along the radii of the walls of steel, copper, aluminum and grey iron circular pipes when the thickness of each wall is approximately: a) $0,136 \mathrm{~cm}, \mathrm{~b}) 0,316$ $\mathrm{cm}, \mathrm{c}) 0,541 \mathrm{~cm}$ and d) $0,766 \mathrm{~cm}\left(r_{2}=1,5\right.$ and internal pressure) 


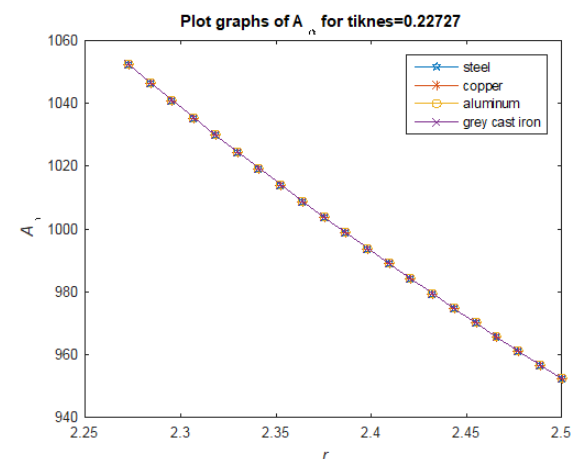

(a)

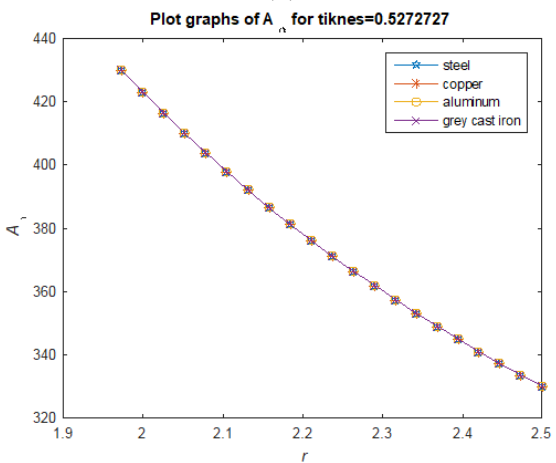

(b)

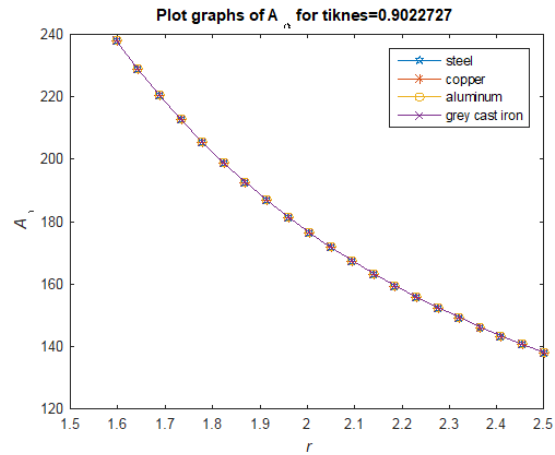

(c)

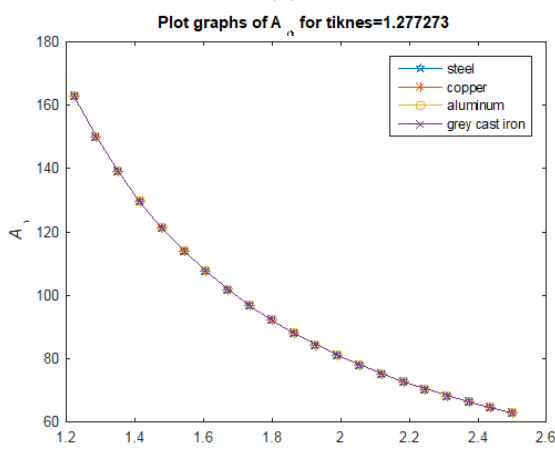

(d)

Fig.6. Shearing stresses $A_{\alpha}$ along the radii of the walls of steel, copper, aluminum and grey iron circular pipes when the thickness of each wall is approximately: a) $0,227 \mathrm{~cm}$, b) 0,527 $\mathrm{cm}, \mathrm{c}) 0,902 \mathrm{~cm}$ and d) $1,277 \mathrm{~cm}\left(r_{2}=2,5\right.$ and internal pressure)

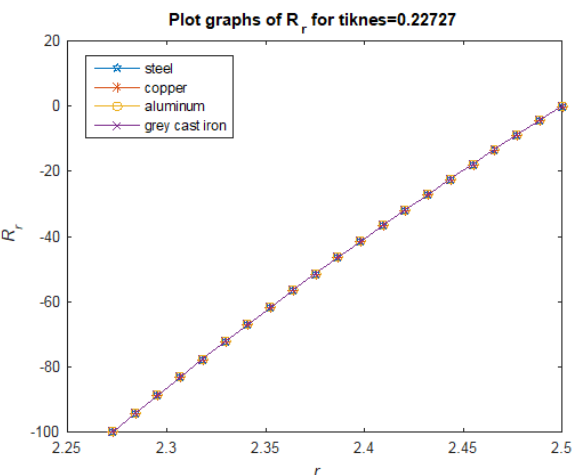

(a)

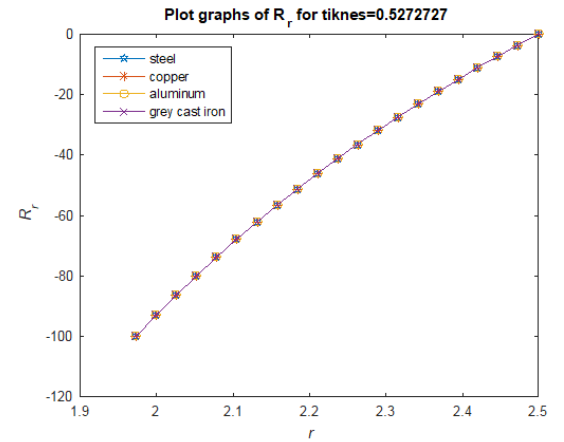

(b)

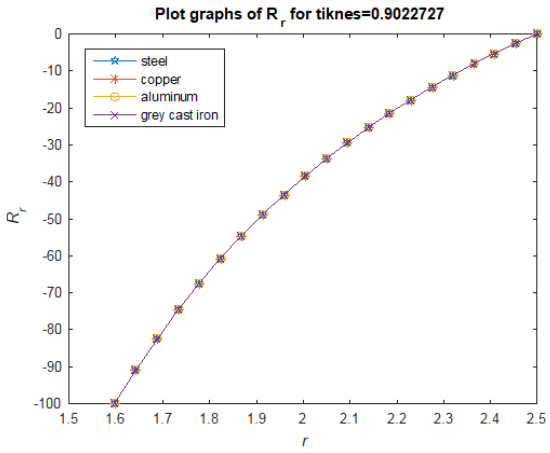

(c)

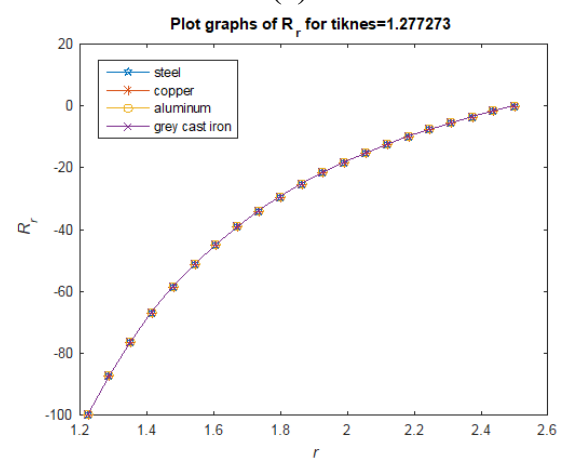

(d)

Fig.7. Normal stresses $R_{r}$ along the radii of the walls of steel, copper, aluminum and grey iron circular pipes when the thickness of each wall is approximately: a) 0,227 cm, b) 0,527 $\mathrm{cm}, \mathrm{c}) 0,902 \mathrm{~cm}$ and d) $1,277 \mathrm{~cm}\left(r_{2}=2,5\right.$ and internal pressure) 


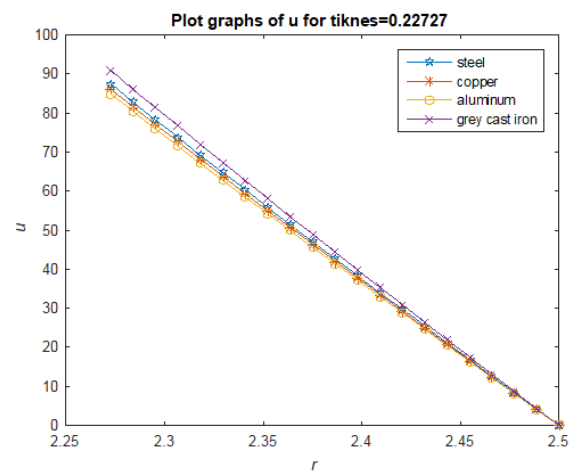

(a)

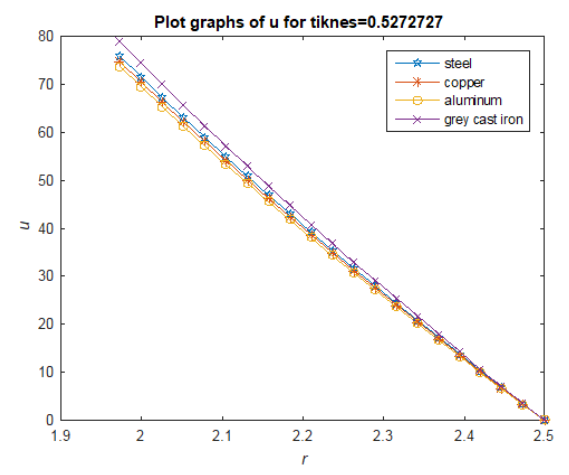

(b)

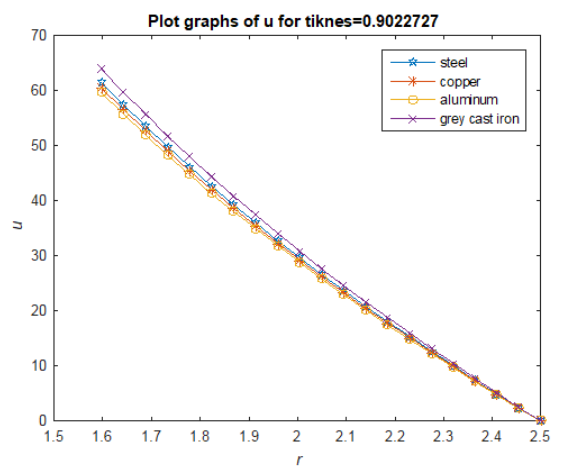

(c)

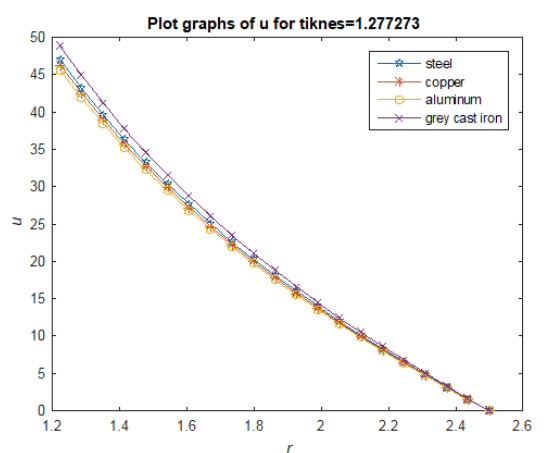

(d)

Fig. 8. Normal displacements $u$ along the radii of the walls of steel, copper, aluminum and grey iron circular pipes when the thickness of each wall is approximately: a) $0,227 \mathrm{~cm}$, b) 0,527 $\mathrm{cm}, \mathrm{c}) 0,902 \mathrm{~cm}$ and d) $1,277 \mathrm{~cm}\left(r_{2}=2,5\right.$ and internal pressure)

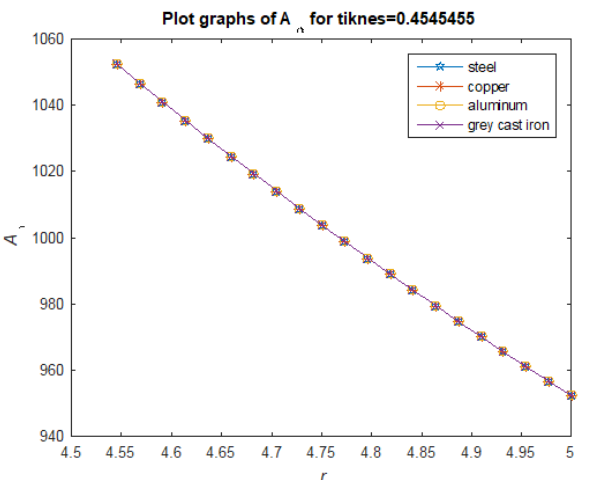

(a)

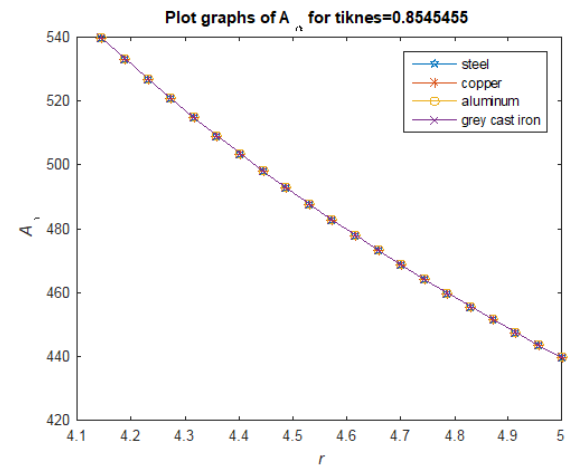

(b)

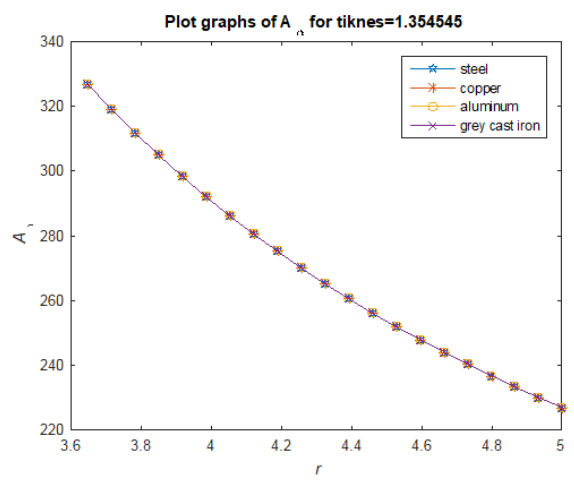

(c)

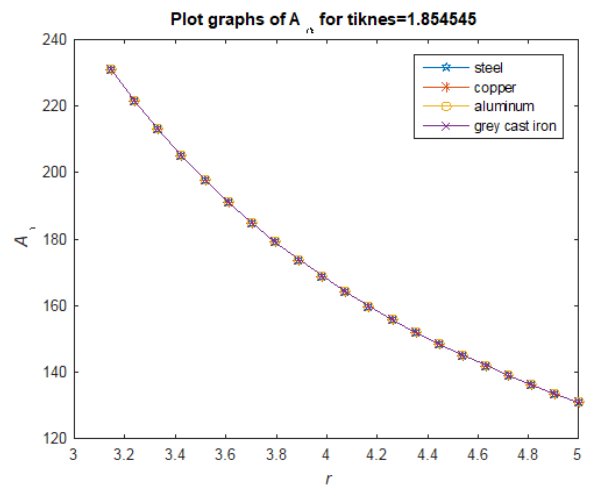

(d)

Fig. 9. Shearing stresses $A_{\alpha}$ along the radii of the walls of steel, copper, aluminum and grey iron circular pipes when the thickness of each wall is approximately: a) $0,454 \mathrm{~cm}$, b) 0,854 $\mathrm{cm}, \mathrm{c}) 1,354 \mathrm{~cm}$ and d) $1,854 \mathrm{~cm}$ ( $r_{2}=5$ and internal pressure) 


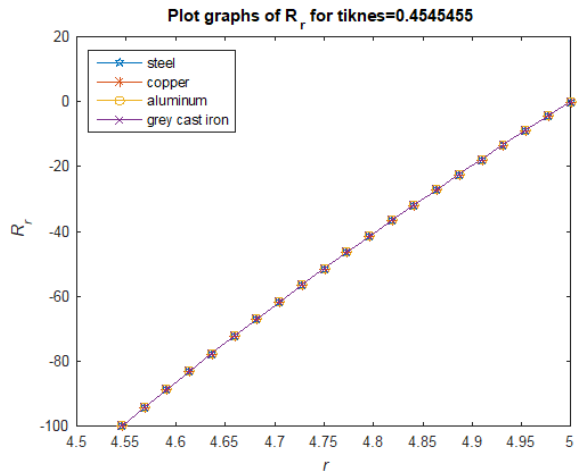

(a)

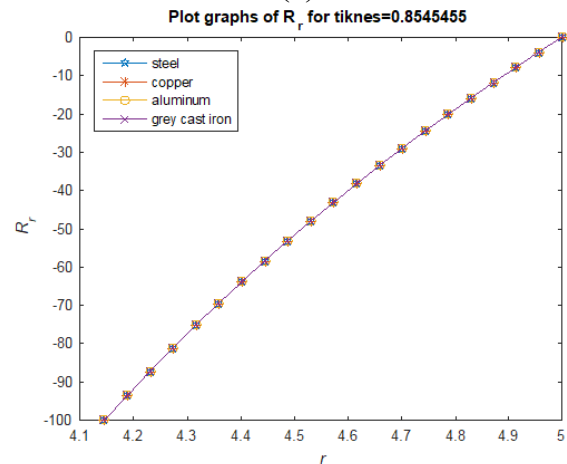

(b)

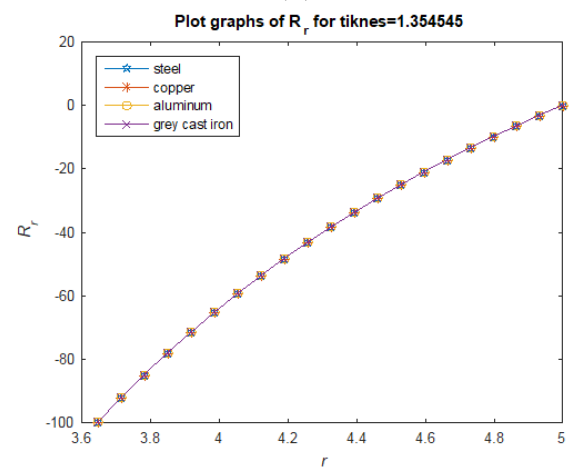

(c)

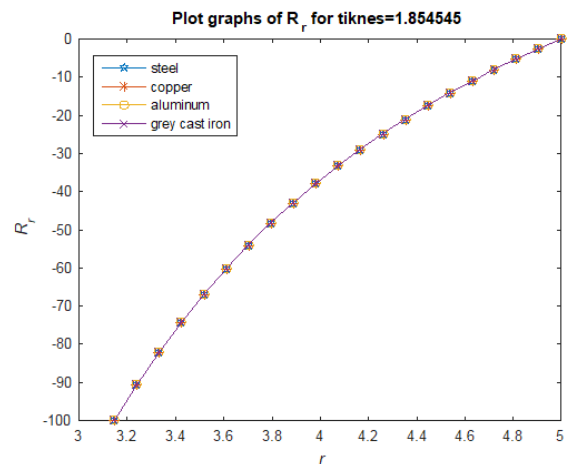

(d)

Fig. 10. Normal stresses $R_{r}$ along the radii of the walls of steel, copper, aluminum and grey iron circular pipes when the thickness of each wall is approximately: a) $0,454 \mathrm{~cm}$, b) 0,854 $\mathrm{cm}, \mathrm{c}) 1,354 \mathrm{~cm}$ and d) $1,854 \mathrm{~cm}\left(r_{2}=5\right.$ and internal pressure $)$

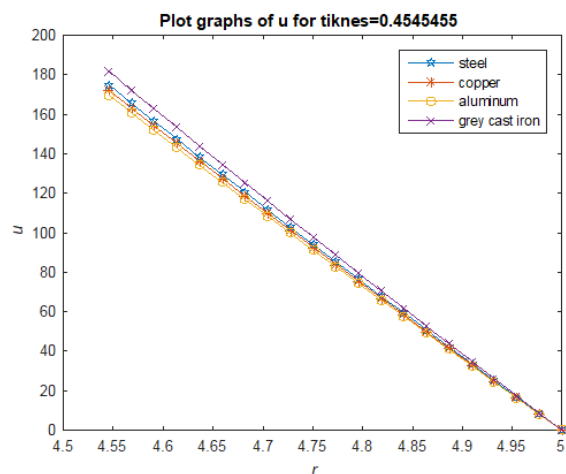

(a)

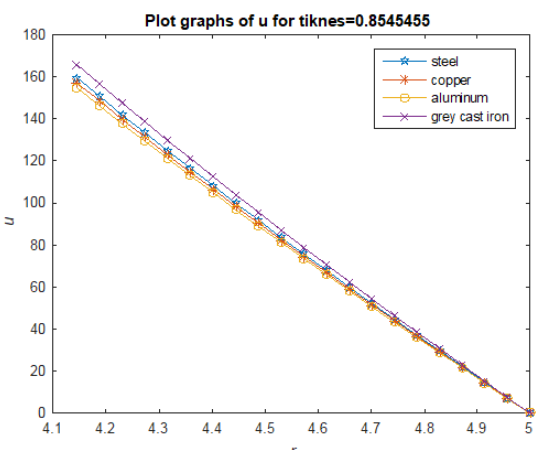

(b)

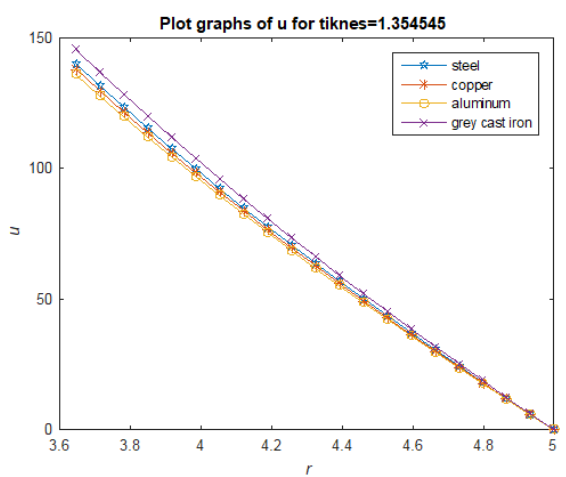

(c)

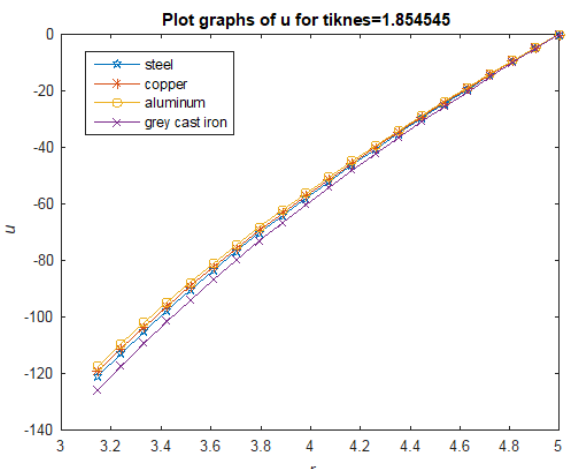

(d)

Fig. 11. Normal displacements $u$ along the radii of the walls of steel, copper, aluminum and grey iron circular pipes when the thickness of each wall is approximately: a) $0,454 \mathrm{~cm}, \mathrm{~b}) 0$, $854 \mathrm{~cm}, \mathrm{c}) 1,354 \mathrm{~cm}$ and d) $1,854 \mathrm{~cm}\left(r_{2}=5\right.$ and internal pressure) 


\section{B. Strength calculation of a cylinder under the impact of external forces}

Let us insert $F_{11}^{(1)}(\alpha)=0, \quad F_{12}^{(1)}(\alpha)=0, \quad F_{11}^{(2)}(\alpha)=-p$, $F_{12}^{(2)}(\alpha)=0$ in formulas (15). We will gain the following system of equations to $b_{10}, b_{20}, A_{1 k}$ and $B_{1 k}$ :

$$
\begin{aligned}
& -4 \mu b_{10}-\frac{2 \mu}{r_{1}^{2}} b_{20}=0, \\
& -n L_{4}\left(\frac{r_{1}}{r_{2}}\right)^{n} A_{1 k}-n L_{3} B_{1 k}-L_{3} \frac{1}{r_{1}^{2}}\left(\frac{r_{1}}{r_{2}}\right)^{n} A_{2 k}+L_{4} \frac{1}{r_{1}^{2}} B_{2 k}=0, \\
& -4 \mu b_{10}-\frac{2 \mu}{r_{2}^{2}} b_{20}=-p, \\
& -n L_{4} A_{1 k}-n L_{3}\left(\frac{r_{1}}{r_{2}}\right)^{n} B_{1 k}-L_{3} \frac{1}{r_{2}^{2}} A_{2 k}+L_{4} \frac{1}{r_{2}^{2}}\left(\frac{r_{1}}{r_{2}}\right)^{n} B_{2 k}=0, \\
& k=1,2, \ldots, \quad n=2 k,
\end{aligned}
$$

where: $\quad L_{1}:=2 \mu n[n(n-1)-2], \quad L_{2}:=2 \mu n[n(n+1)-2]$, $L_{3}:=2 \mu n(n-1), \quad L_{4}:=2 \mu n(n+1), \quad n=2 k$.

From here:

$$
\begin{aligned}
& b_{10}=\frac{p r_{2}^{2}}{4 \mu\left(r_{2}^{2}-r_{1}^{2}\right)}, \\
& b_{20}=-\frac{p r_{1}^{2} r_{2}^{2}}{2 \mu\left(r_{2}^{2}-r_{1}^{2}\right)} . \\
& A_{i k}=0, \quad B_{i k}=0, \quad i=1,2, \quad k=1,2, \ldots . \\
& A_{\alpha}=-4 \mu b_{10}+\frac{2 \mu}{r^{2}} b_{20}
\end{aligned}
$$

After inserting expression (21) in equations (13) and (14), the following expressions are obtained:

$$
\begin{gathered}
u=-\frac{1}{2(\lambda+\mu)} r \frac{p r_{2}^{2}}{r_{2}^{2}-r_{1}^{2}}-\frac{1}{2 \mu} \frac{1}{r} \frac{p r_{1}^{2} r_{2}^{2}}{r_{2}^{2}-r_{1}^{2}} . \\
R_{r}=-\frac{p r_{2}^{2}}{r_{2}^{2}-r_{1}^{2}}+\frac{r_{1}^{2} r_{2}^{2}}{r^{2}} \frac{p}{r_{2}^{2}-r_{1}^{2}}, \\
A_{\alpha}=-\frac{p r_{2}^{2}}{r_{2}^{2}-r_{1}^{2}}-\frac{1}{r^{2}} \frac{p r_{1}^{2} r_{2}^{2}}{r_{2}^{2}-r_{1}^{2}}, \\
R_{\alpha}=0 .
\end{gathered}
$$

Displacements and stresses at any point of the considered area are obtained from equations (22) and (23).

\begin{tabular}{|c|c|c|c|c|c|c|}
\hline \multirow{2}{*}{ Material } & \multicolumn{3}{|c|}{$\begin{array}{l}\text { Minimum admissible wall thickness } \\
\left(r_{2}-r_{1}\right), \mathrm{cm}\end{array}$} & \multirow{2}{*}{$\begin{array}{l}E \\
\mathrm{Kg} / \mathrm{cm}^{2}\end{array}$} & \multirow[b]{2}{*}{$v$} & \multirow{2}{*}{$\begin{array}{l}\text { Admis } \\
\text { ible } \\
\text { stress, } \\
\mathrm{Kg} / \mathrm{cm}\end{array}$} \\
\hline & $r_{2}=1.5 \mathrm{~cm}$ & $r_{2}=2.5 \mathrm{~cm}$ & $r_{2}=5 \mathrm{~cm}$ & & & \\
\hline Steel & 0.2263636 & 0.3772727 & 0.7545455 & $2 \cdot 10^{6}$ & 0.3 & 713.801 \\
\hline Copper & 0.2713636 & 0.4522727 & 0.8545455 & $1.1 \cdot 10^{6}$ & 0.32 & 611.830 \\
\hline \begin{tabular}{|l|} 
Aluminum \\
\end{tabular} & 0.3163636 & 0.5272727 & 1.054545 & $0.7 \cdot 10^{6}$ & 0.34 & 509.858 \\
\hline \begin{tabular}{|l|} 
Grey cast \\
iron
\end{tabular} & 0.3163636 & 0.5272727 & 1.054545 & $0.7 \cdot 10^{6}$ & 0.25 & 509.858 \\
\hline
\end{tabular}

If we take $p_{1}=0, \quad p_{2}=p$ in (16), (17), then expressions (16), (17) will be given as (22) and (23).

By using (16), (17) formulas, or formulas (22) and (23), we obtain the minimum wall thickness of a circular steel, copper, aluminum and grey cast iron ring, at which the stresses produced in the body do not exceed the admissible value, when $r_{2}=1.5,2.5,5$. The obtained numerical values are presented in table 2 .
TABLE II. Minimum admissible thickness of a cylinder wall at which the stresses produced in the cylinders of different materials and diameters do not exceed admissible values (external load)

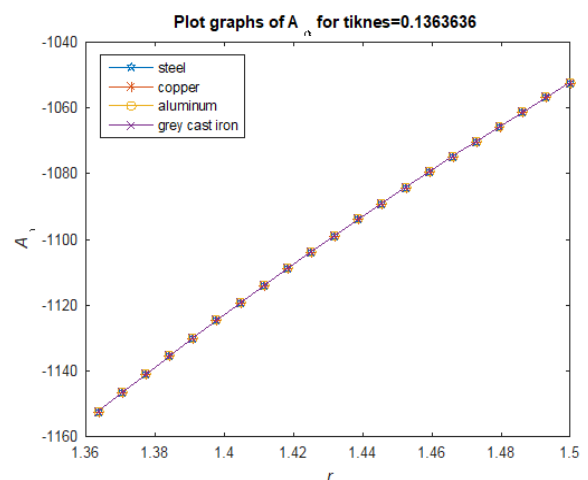

(a)

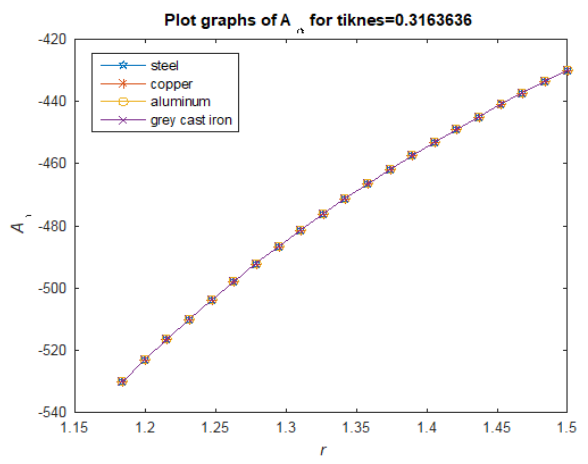

(b)

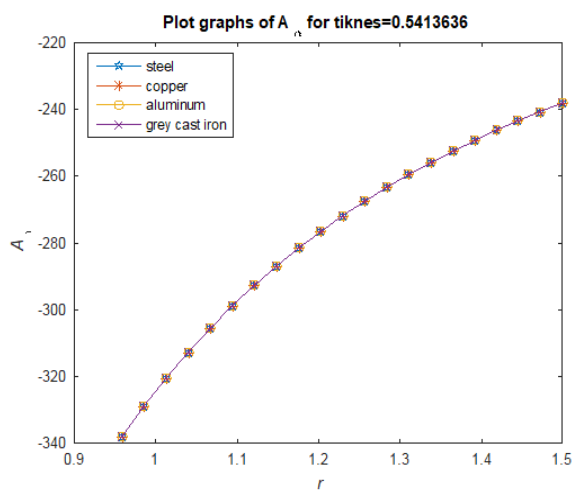

(c) 


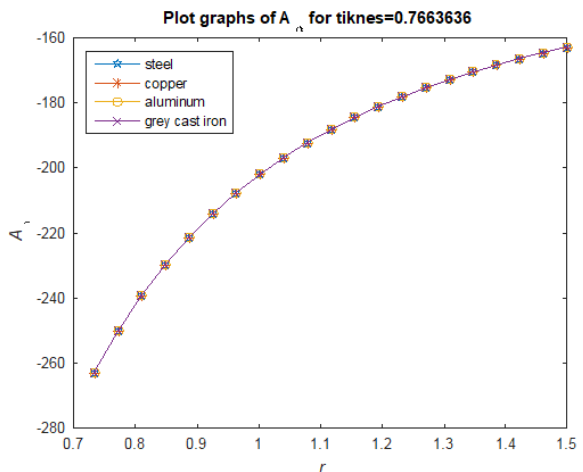

(d)

Fig. 12. Shearing stresses $A_{\alpha}$ along the radii of the walls of steel, copper, aluminum and grey iron circular pipes when the thickness of each wall is approximately: a) $0,136 \mathrm{~cm}, \mathrm{~b}) 0,316$ $\mathrm{cm}, \mathrm{c}) 0,541 \mathrm{~cm}$ and d) $0,766 \mathrm{~cm}\left(r_{2}=1,5\right.$ and external

pressure)

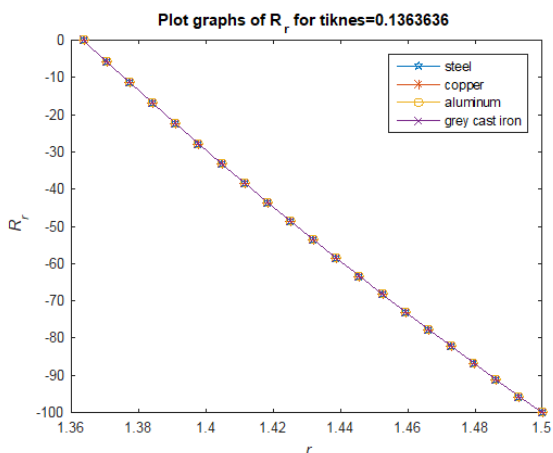

(a)

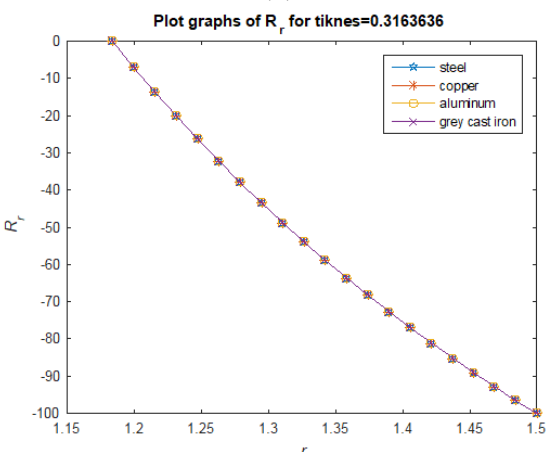

(b)

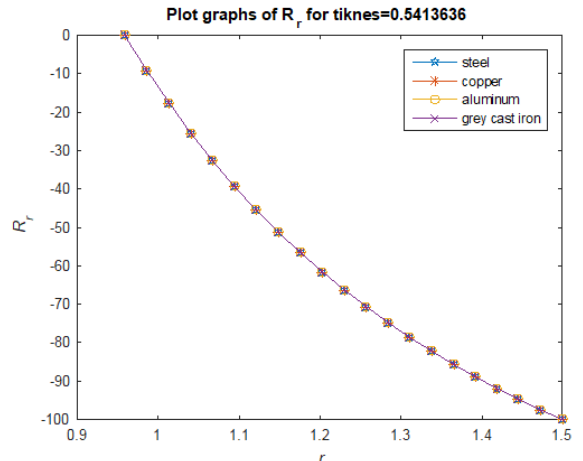

(c)

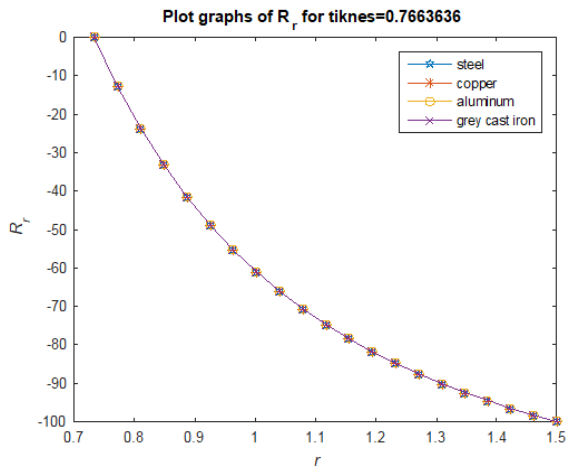

(d)

Fig. 13 Normal stresses $R_{r}$ along the radii of the walls of steel, copper, aluminum and grey iron circular pipes when the thickness of each wall is approximately: a) $0,136 \mathrm{~cm}, \mathrm{~b}) 0,316$ $\mathrm{cm}, \mathrm{c}) 0,541 \mathrm{~cm}$ and d) $0,766 \mathrm{~cm}\left(r_{2}=1,5\right.$ and external

pressure)

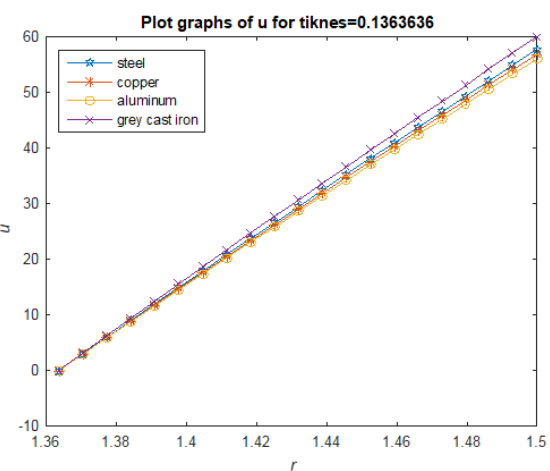

(a)

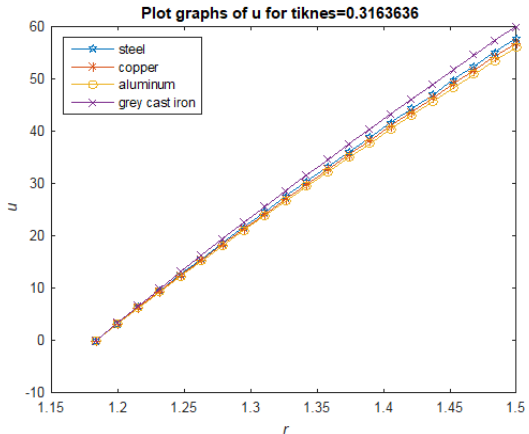

(b)

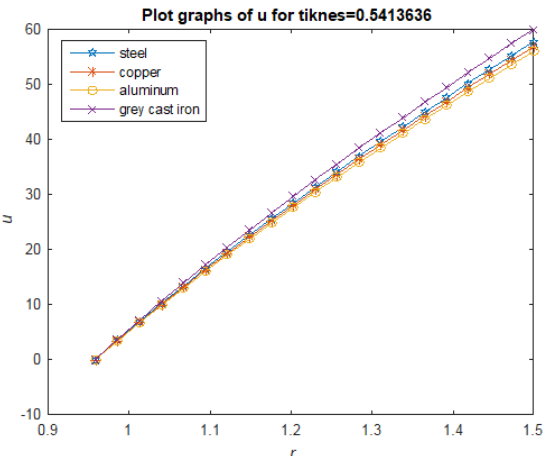

(c) 


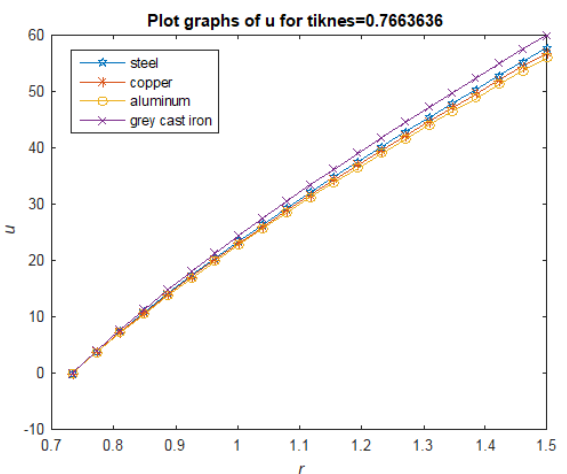

(d)

Fig. 14 Normal displacements $u$ along the radii of the walls of steel, copper, aluminum and grey iron circular pipes when the thickness of each wall is approximately: a) $0,136 \mathrm{~cm}$, b) 0,316 $\mathrm{cm}, \mathrm{c}) 0,541 \mathrm{~cm}$ and d) $0,766 \mathrm{~cm}\left(r_{2}=1,5\right.$ and external

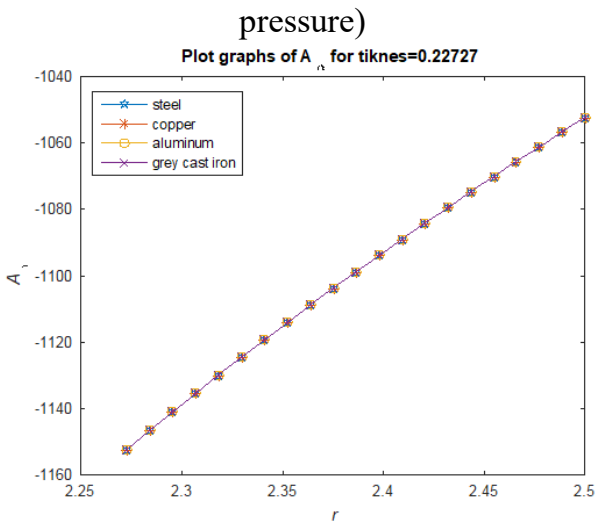

(a)

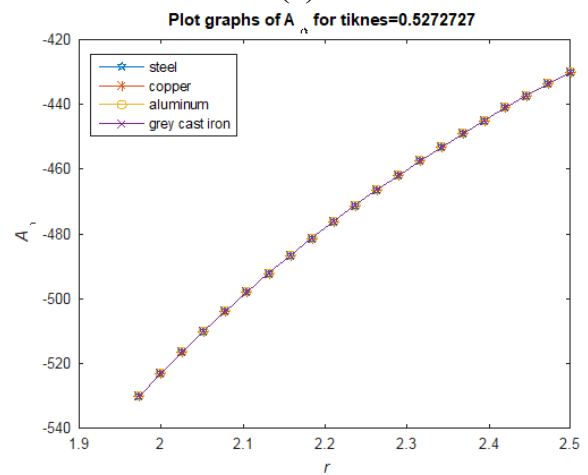

(b)

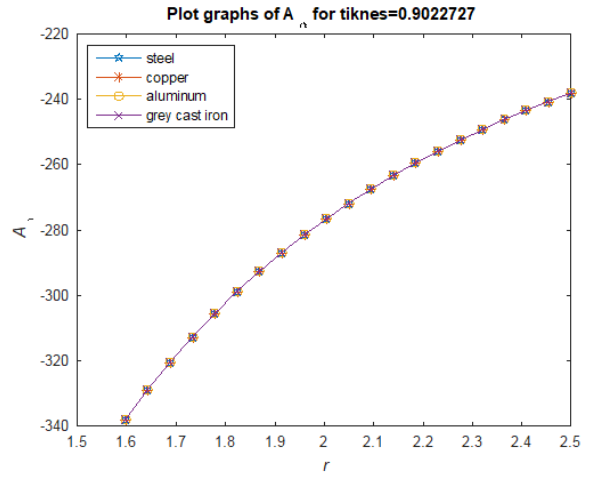

(c)

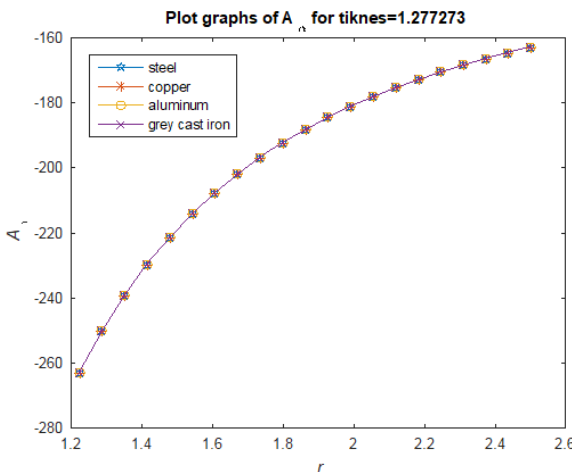

(d)

Fig. 15 Shearing stresses $A_{\alpha}$ along the radii of the walls of steel, copper, aluminum and grey iron circular pipes when the thickness of each wall is approximately: a) $0,227 \mathrm{~cm}$, b) 0,527 $\mathrm{cm}, \mathrm{c}) 0,902 \mathrm{~cm}$ and d) $1,277 \mathrm{~cm}\left(r_{2}=2,5\right.$ and external

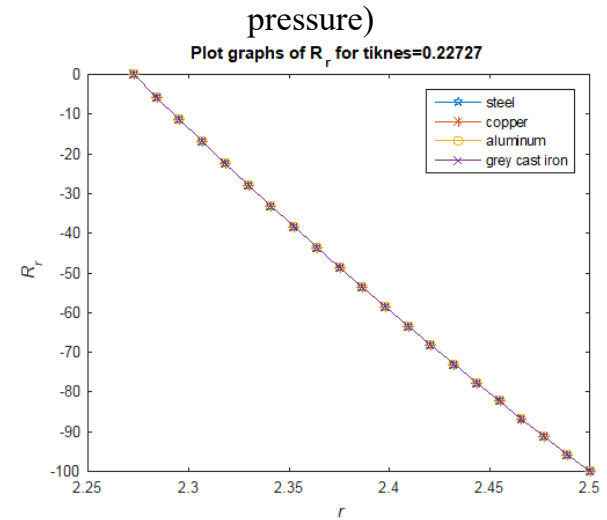

(a)

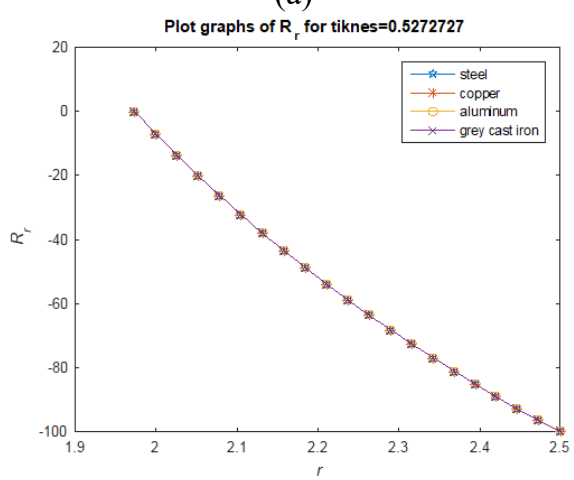

(b)

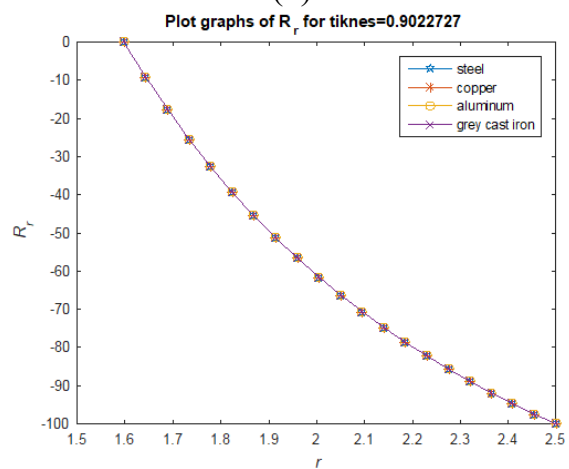

(c) 


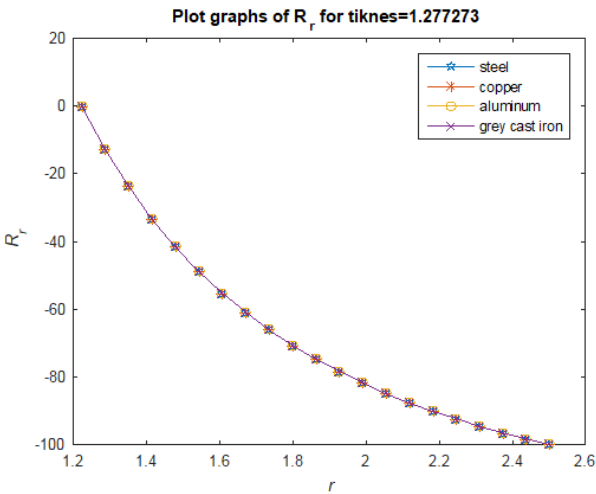

(d)

Fig. 16 Normal stresses $R_{r}$ along the radii of the walls of steel, copper, aluminum and grey iron circular pipes when the thickness of each wall is approximately: a) $0,227 \mathrm{~cm}$, b) 0,527

$\mathrm{cm}, \mathrm{c}) 0,902 \mathrm{~cm}$ and d) $1,277 \mathrm{~cm}\left(r_{2}=2,5\right.$ and external pressure)

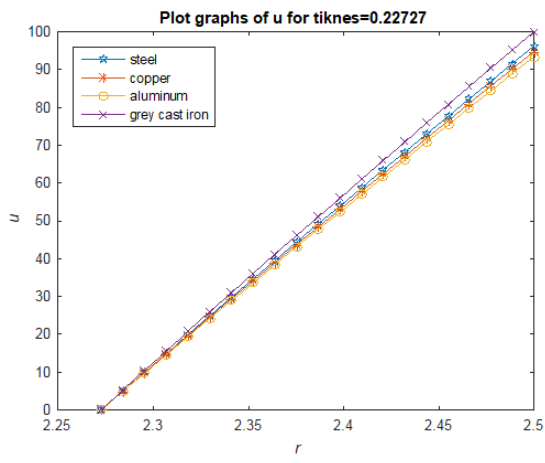

(a)

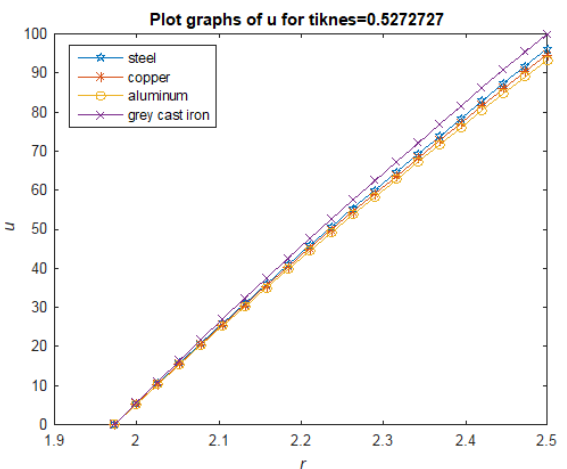

(b)

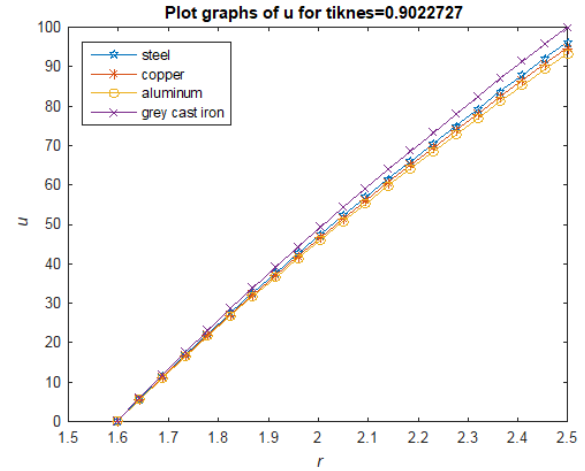

(c)

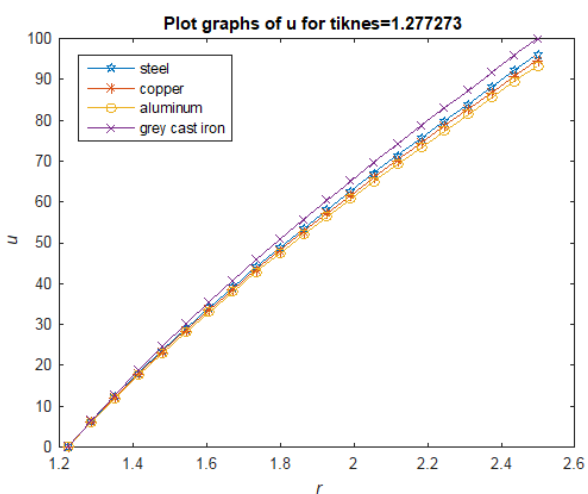

(d)

Fig. 17 Normal Displacements $u$ along the radii of the walls of steel, copper, aluminum and grey iron circular pipes when the thickness of each wall is approximately: a) $0,227 \mathrm{~cm}$, b) 0 , $527 \mathrm{~cm}, \mathrm{c}) 0,902 \mathrm{~cm}$ and d) $1,277 \mathrm{~cm}\left(r_{2}=2,5\right.$ and external

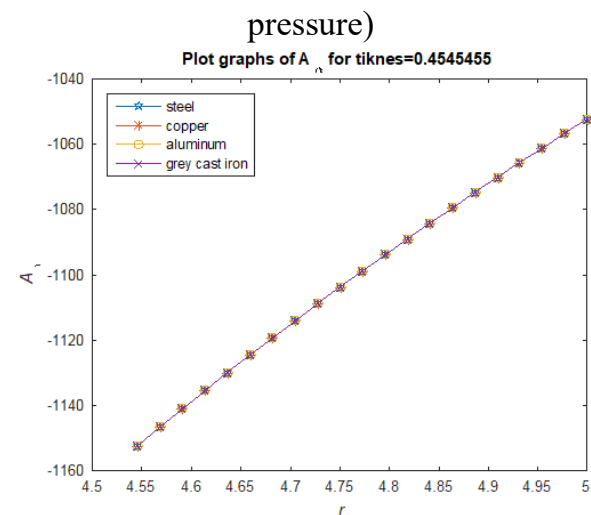

(a)

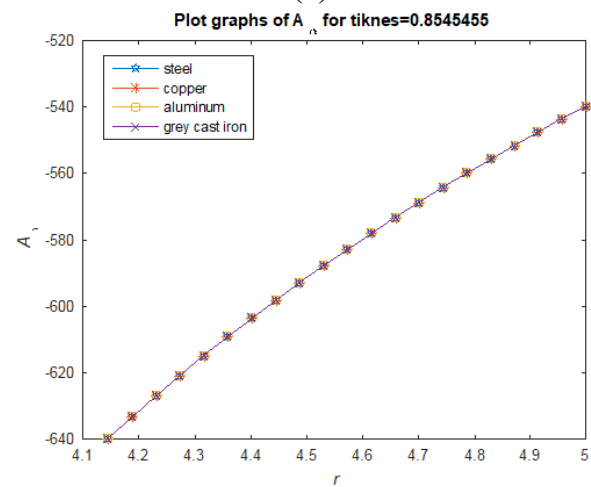

(b)

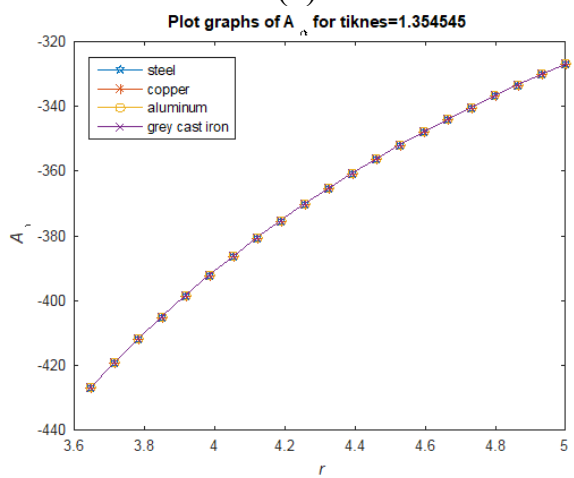

(c) 


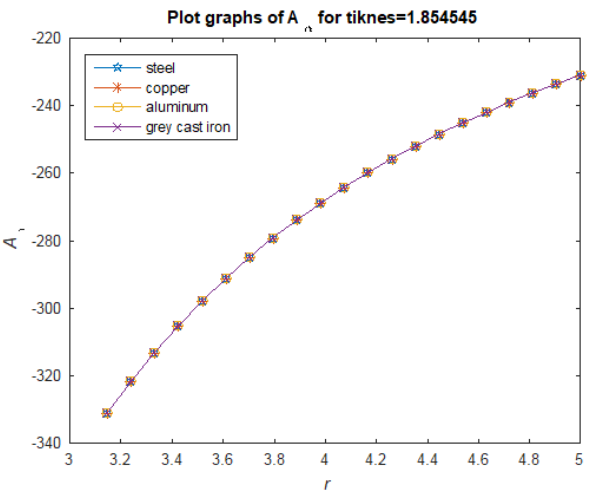

(d)

Fig. 18 Shearing stresses $A_{\alpha}$ along the radii of the walls of steel, copper, aluminum and grey iron circular pipes when the thickness of each wall is approximately: a) $0,454 \mathrm{~cm}$, b) 0,854 $\mathrm{cm}, \mathrm{c}) 1,354 \mathrm{~cm}$ and d) $1,854 \mathrm{~cm}\left(r_{2}=5\right.$ and external

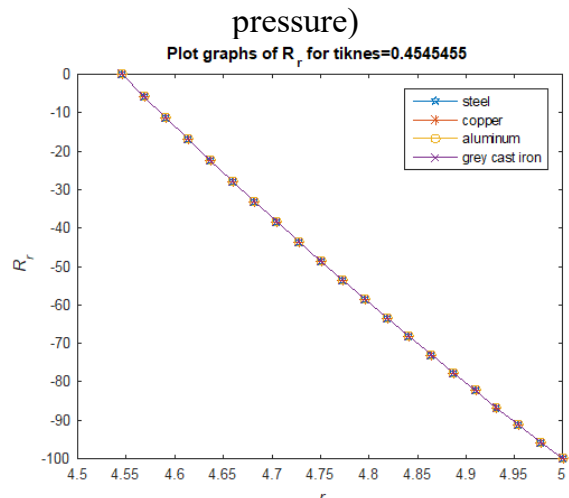

(a)

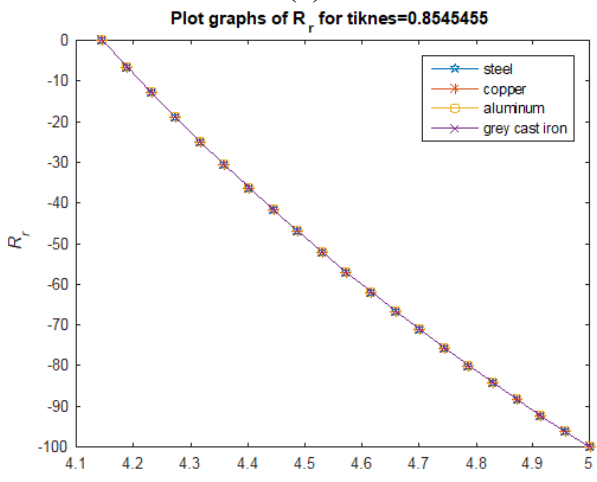

(b)

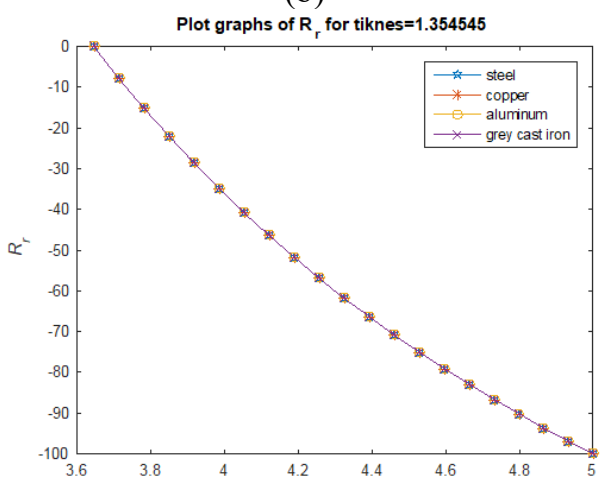

(c)

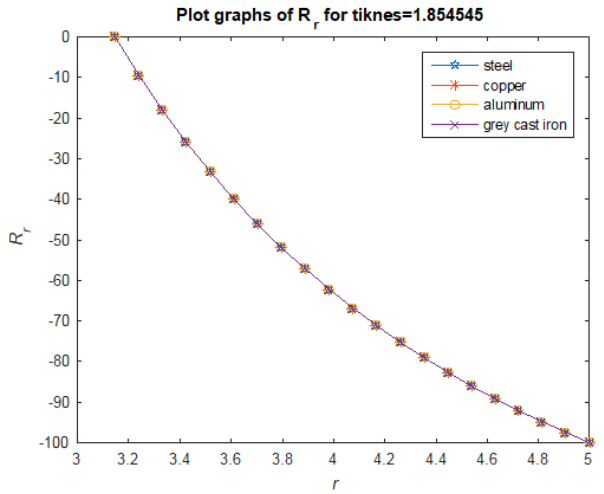

(d)

Fig. 19 Normal stresses $R_{r}$ along the radii of the walls of steel, copper, aluminum and grey iron circular pipes when the thickness of each wall is approximately: a) $0,454 \mathrm{~cm}, \mathrm{~b}) 0,854$ $\mathrm{cm}$, c) $1,354 \mathrm{~cm}$ and d) $1,854 \mathrm{~cm}$ ( $r_{2}=5$ and external pressure)

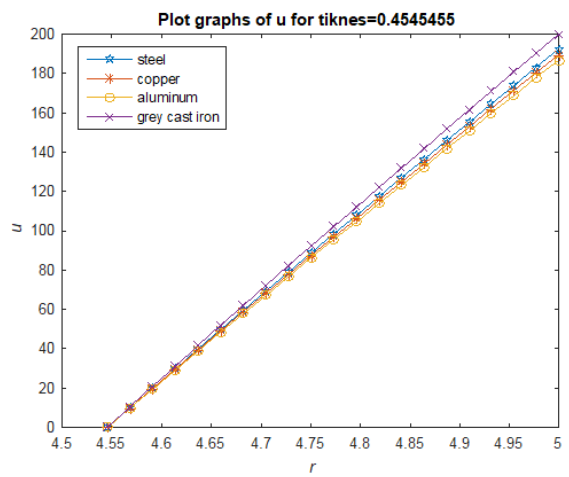

(a)

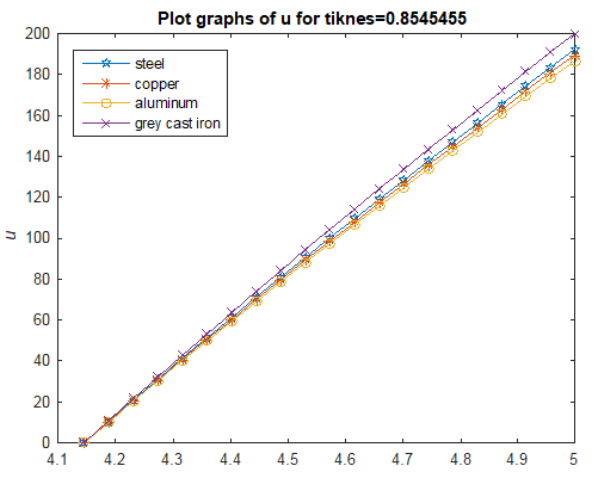

(b)

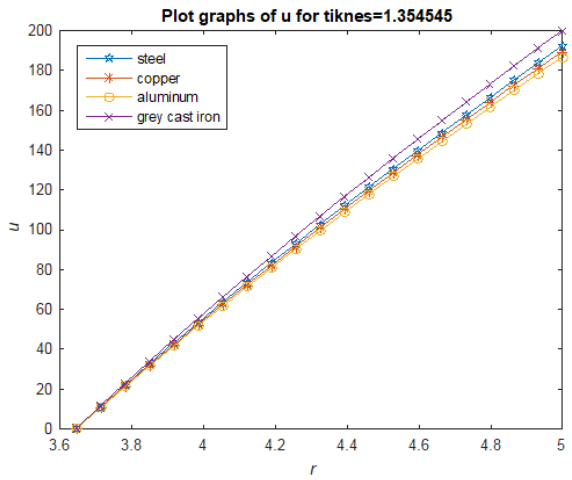

(c) 


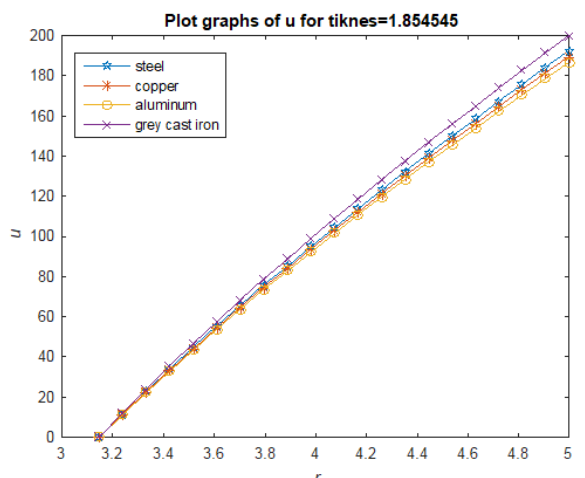

(d)

Fig. 20 Normal displacements $u$ along the radii of the walls of steel, copper, aluminum and grey iron circular pipes when the thickness of each wall is approximately: a) $0,454 \mathrm{~cm}, \mathrm{~b}) 0$, $854 \mathrm{~cm}, \mathrm{c}) 1,354 \mathrm{~cm}$ and d) $1,854 \mathrm{~cm}\left(r_{2}=5\right.$ and external

$$
\text { pressure) }
$$

\section{Consideration of results}

As it was expected and as the tables show, the minimum admissible wall thickness of a steel pipe (cylinder) is less than that of a copper pipe, while the minimum admissible wall thickness of a copper pipe is even less than that of an aluminum pipe. As the Young modulus values for aluminum and grey cast iron are the same, the minimum admissible thicknesses of their walls are also the same. It should also be noted that the greater the pipe diameter is, the more the minimum admissible wall thickness is.

The tables show the minimum thicknesses of a ring (cylinder wall), when the stresses produced in the pipe of different materials (steel, copper, aluminum and grey cast iron) and different diameters $(3 \mathrm{~cm}, 5 \mathrm{~cm}$ and $10 \mathrm{~cm})$ do not exceed the relevant admissible values, when (1) constant normal stress is applied to the internal boundary of a ring and the external boundary is free from stresses (Table 1), or (2) constant normal stress is applied to the external boundary and the internal boundary is free from stress (Table 2). These tables show that the minimum admissible pipe wall thickness with the loaded internal boundary is less than with the loaded external boundary.

Normal and shearing stresses and normal displacements along the radius in different-thickness walls of steel, copper, aluminum and grey iron pipes under the influence of internal pressures are given in Fig. 4 - Fig 10 and external pressures are given in Fig. 11 - Fig. 18. The obtained numerical results and relevant graphs give the following picture: (a) the pressures and displacements decrease as the wall thickness increases, and (b) in the case considered in the given article (i.e. under the permanent normal load), the pressures do not depend on the material (elastic properties) what is also seen from the formulas, in particular, see (17), while greatest displacements are seen with grey iron pipes and the smallest ones are seen with aluminum pipes, (c) normal displacements are greater in case of external pressure than with internal pressure, (d) in case of internal pressure, shearing stresses have a positive direction (positive numerical value), while in case of external pressure, they have a negative direction (negative value).

\section{CONCLUSION}

In Systems Theory, the Mathematical and numerical simulation of strength of thick-wall pipe by using static elastic problems is an important problem and has attracted the attention of many researches, academicians and practitioners. The principle results presented in the present work can be formulated as follows:

- The strength of quite a long homogeneous isotropic thick-walled circular pipe (cylinder) under the impact of external forces (internal or external pressure) by using the problems of statics of the theory of elasticity is studied. Plane deformation is considered.

- Mathematical and numerical modeling is provided to study the strength of a thick-walled circular pipe under the impact of external forces (internal or external pressure) by using the problems of statics of the theory of elasticity.

- The problems of statics of the theory of elasticity are set and solved in the polar coordinate system.

- An analytical solution is obtained by using the method of separation of variables, which is presented as two harmonic functions.

- Some numerical results are given and considered and graphs of normal and shearing stresses and normal displacements along the radii in the walls with different thicknesses of steel, copper, aluminum and grey iron pipes under the influence of internal and external pressures are drafted.

- The minimum wall thicknesses of the pipes of different materials (steel, copper, aluminum and grey cast iron namely) and diameters $(3 \mathrm{~cm}, 5 \mathrm{~cm}$ and $10 \mathrm{~cm}$ namely), when the values of stresses originated in them do not exceed the admissible values, are identified.

A cylindrical vessel under the impact of stress is often used as a component in such branches of industry as chemical, military and oil industries and water and nuclear power plants. These components are often subject to a complex load, such as twisting, pressure, temperature, etc. The circular cylinders (pipes) are also widely used in building, machine building, etc. Therefore, the study of the deflected mode of the cylindrical bodies is topical and so, in my opinion, setting the problems considered in the present work and the method of their solution is interesting in a practical view. 


\section{APPENDIX A}

Theorem 2 If function $\varphi$ is harmonic, then $(r \varphi)_{, r}$ is a harmonic function.

Proof Let us assume that $\Delta \varphi=0$. We must prove that $\Delta(r \varphi)_{, r}=0 \quad(\Delta($.$) \quad (is a Laplace operator), i.e.$ $L:=\Delta\left(r \varphi_{, r}+\varphi\right)=0$. So, we must prove that:

$$
L=r \varphi_{, r r r}+3 \varphi_{, r r}+\frac{1}{r} \varphi_{, r}+\frac{1}{r} \varphi_{, r \alpha \alpha}=0 .
$$

As $\Delta \varphi=0$, then $r(\Delta \varphi)_{, r}=0 ; r\left(\varphi_{, r r}+\frac{1}{r} \varphi_{, r}+\frac{1}{r^{2}} \varphi_{, \alpha \alpha}\right)_{, r}=0$. i.e.

$$
r \varphi_{, r r r}+\varphi_{, r r}-\frac{1}{r} \varphi_{, r}+\frac{1}{r} \varphi_{, r \alpha \alpha}-\frac{2}{r^{2}} \varphi_{, \alpha \alpha}=0
$$

Let us write down operator $L$ as follows:

$$
\begin{aligned}
L= & \underline{r \varphi_{, r r r}}+\underline{\varphi_{, r r}}-\frac{1}{r} \varphi_{, r}+2 \varphi_{, r r}+\frac{2}{r} \varphi_{, r}+\frac{1}{r} \varphi_{, r \alpha \alpha} \\
& +\frac{2}{r^{2}} \varphi_{, \alpha \alpha}-\frac{2}{\underline{r^{2}}} \varphi_{, \alpha \alpha}=0 .
\end{aligned}
$$

The underlined members in (A.2), by the virtue of (A.1), give zero, while other three members give a Laplace operator, which equals to zero. Thus, $L=0$, i.e. $\Delta(r \varphi)_{, r}=\Delta\left(r \varphi_{, r}+\varphi\right)=0$, i.e. $(r \varphi)_{, r}$ is harmonic. Thus, the theorem is proved.

\section{APPENDIX B: PROVING SOME FORMULAS}

Let us assume that $\varphi$ is a harmonic function, i.e. $\Delta \varphi=0$. It is easy to prove that:

1) $r\left(r \varphi_{1}\right)_{, r r}=\left(r^{2} \varphi_{1, r}\right)_{, r}$;

2) $(r \varphi)_{, r}=\Delta\left(\frac{r^{2}}{4} \varphi\right)$;

3) $\left(r^{2} \varphi_{, r}\right)_{, r}=\Delta\left(\frac{r^{3}}{4} \varphi_{, r}\right)$.

Proof

1) $r \kappa_{1, r}+r\left(r \varphi_{1, r}\right)_{, r}=2 r \varphi_{1, r}+r^{2} \varphi_{1, r r}=\left(r^{2} \varphi_{1, r}\right)$.

2)

$$
\begin{aligned}
\Delta\left(\frac{r^{2}}{4} \varphi\right) & =\frac{1}{2} \varphi+r \varphi_{, r}+\frac{r^{2}}{4} \varphi_{, r r}+\frac{1}{2} \varphi \\
+ & \frac{r}{4} \varphi_{, r}+\frac{1}{4} \varphi_{, \alpha \alpha}=\varphi+r \varphi_{, r}=(r \varphi)_{, r} .
\end{aligned}
$$

The sum of the underlined members is a Laplace operator in a polar coordinate system and as $\varphi$ is a harmonic function, it equals to zero.

3) $\Delta\left(\frac{r^{3}}{4} \varphi_{, r}\right)=\frac{9 r}{4} \varphi_{, r}+\frac{3 r^{2}}{2} \varphi_{, r r}+\frac{r^{3}}{4} \varphi_{, r r r}+\frac{r^{2}}{4} \varphi_{, r r}+\frac{r}{4} \varphi_{, r \alpha \alpha}$;

$$
\begin{aligned}
\frac{r^{3}}{4}(\Delta \varphi)_{, r}= & 0 \text { as } \Delta \varphi=0 . \\
\frac{r^{3}}{4}(\Delta \varphi)_{, r}= & \frac{r^{3}}{4}\left(\varphi_{, r r}+\frac{1}{r} \varphi_{, r}+\frac{1}{r^{2}} \varphi_{, \alpha \alpha}\right)=\frac{r^{3}}{4} \varphi_{, r r}+\frac{r^{2}}{4} \varphi_{, r r}-\frac{r}{4} \varphi_{, r} \\
& +\frac{r}{4} \varphi_{, r \alpha \varepsilon}-\frac{1}{2} \varphi_{, \alpha \alpha}=0 .
\end{aligned}
$$

By considering this, we will gain:

$$
\begin{aligned}
\Delta\left(\frac{r^{3}}{4} \varphi_{, r}\right) & =\frac{5 r}{4} \varphi_{, r}+\frac{3 r^{2}}{2} \varphi_{, r r}+\frac{1}{2} \varphi_{, \alpha \alpha} \\
& =\frac{1}{2}\left[\left(4 r \varphi_{, r}+2 r^{2} \varphi_{, r r}\right)+\left(r^{2} \varphi_{, r r}+r \varphi_{, r}+\varphi_{, \alpha \alpha}\right)\right]= \\
& =2 r \varphi_{, r}+r^{2} \varphi_{, r r}=\left(r^{2} \varphi_{, r}\right)_{, r} .
\end{aligned}
$$

This is what we wanted to prove.

\section{APPENDIX C: SOLVING LAPLACE EQUATIONS IN A POLAR COORDINATE SYSTEM}

Let us solve the following equation:

$$
\varphi_{, r r}+\frac{1}{r} \varphi_{, r}+\frac{1}{r^{2}} \varphi_{, \alpha \alpha}=0 .
$$

Let us try to find the solution as follows:

$$
\varphi=R(r) A(\alpha) \text {. }
$$

After inserting (C.2) in (C.1), we will obtain:

$$
r^{2} \frac{R^{\prime \prime}}{R}+r \frac{R^{\prime}}{R}+\frac{A^{\prime \prime}}{A}=0
$$

Let us consider two cases:

1)

$r^{2} \frac{R^{\prime \prime}}{R}+r \frac{R^{\prime}}{R}=k^{2}, \quad \frac{A^{\prime \prime}}{A}=-k^{2}, k$ is a real number (the imaginary case is deduced to the real one).

$$
\begin{aligned}
& r^{2} R^{\prime \prime}+r R^{\prime}-k^{2} R=0 \text {; } \\
& R=A_{k} r^{k}+B_{k} r^{-k}+C_{0} \ln r+D_{0} ; \\
& A^{\prime \prime}+k^{2} A=0 \text {; } \\
& A=a_{k} \sin (k \alpha)+b_{k} \cos (k \alpha)+a_{0} \alpha+b_{0} \text {. } \\
& r^{2} \frac{R^{\prime \prime}}{R}+r \frac{R^{\prime}}{R}=-k^{2}, \quad \frac{A^{\prime \prime}}{A}=k^{2} \\
& r^{2} R^{\prime \prime}+r R^{\prime}+k^{2} R=0 ; \\
& \left.R=\widetilde{a}_{k} \sin (k \ln r)\right)+\widetilde{b}_{k} \cos (k \ln r)+\widetilde{C}_{0} \ln r+\widetilde{D}_{0} ; \\
& A^{\prime \prime}-k^{2} A=0 \text {; } \\
& A=\widetilde{A}_{k} e^{k \varepsilon}+\widetilde{B}_{k} e^{-k \alpha}+\widetilde{a}_{0} \alpha+\widetilde{b}_{0} . \\
& \varphi=\left(A_{k} r^{k}+B_{k} r^{-k}\right)_{\cos (k \alpha)}^{\sin (k \alpha)}+\left(\widetilde{A}_{k} e^{\alpha k}+\widetilde{B}_{k} e^{-k \alpha}\right)_{\cos (k \ln r)}^{\sin (k \ln r)} \\
& +a_{0} \alpha \ln r+b_{0} \ln r+c_{0} \alpha+d_{0} .
\end{aligned}
$$

If doing operation $r \varphi_{, r}+\varphi$ for (C.3), the generality will not be violated. If we do the same operation for the first summand of expression $\varphi$, we will gain the summand of the same type, as 
the differentiation with $r$ will not change its type. As a result of differentiating the second summand with $r$ (the expression in brackets does not depend on $r) \sin (k \ln r)$ and $\cos (k \ln r)$

yield $\frac{k}{r} \cos (k \ln r)$ and $-\frac{k}{r} \sin (k \ln r)$, consequently, $r$ will be cancelled, $k$ is constant and will be included in the coefficients. As for other summands, they will be obtained by operation $\varphi$, while $r(.)_{, r}$ will give $a_{0} \alpha$ and $b_{0} \alpha$, which will be united with $c_{0} \alpha$ and $d_{0}$. Thus, neither of the members in expression $\varphi$ is lost or changes its type. So, if we do operation $r \varphi_{, r}+\varphi$ for this sum, the generality will be maintained.

\section{REFERENCES}

[1]A. Ch. Yiannopoulos, A Simplified Solution for Stresses in Thick-Wall Cylinders for Various Loading Conditions, Computers \& Strucrures, 60, 4, 571-578, 1996.

[2]A. Bagheri, D. Taghizadeh, H. Darijani, On the behavior of rotating thick-walleded cylinders made of hyperelastic materials, Meccanica, 51, 3, 973-692, 2016.

[3] C. Changfu, X. Shujun, Y.Yu. Unified, Solution of limit loads of thick wall cylinder subject to external pressure considering strain softenin,. Front. Archit. Civ. Eng. China, 1, 3, 329-333, 2007.

[4]R. Reghunath, S. Korah, Analysis of Internally Pressurized Thick Walled Cylinders, Journal of Basic and Applied Engineering Research, 1, 2, 88-93, 2014.

[5] S. Sharma, S. Yadav, R. Sharma, Creep torsion in thick-walleded circular cylinder under internal and external pressure. Structural Integrity and Life, 18, 2, 89-97, 2018.

[6]R.W. Bailey, Steam piping for high pressures and high temperatures, Proceedings of the Institution of Mechanical Engineers, 164, 1, 324-350, 1951.

[7]F.K.G. Odquist, Mathematical theory of creep and creep rupture, Carendon Press, Oxford, 1974.

[8]R.H. King, W.W. Mackie, Creep of thick-walled cylinders, Journal of Basic Engineering, 89, 877884, 1967.

[9]S.K. Gupta, Elastic-plastic and creep transition of thick-walled cylinder under uniform pressure, Proc. Int. Symp. Non-linear Mech., Kharagpur, 169-175, 1980.

[10] S.K. Gupta, R. L. Dharmani, Creep transition in bending of rectangular plates, Int. J. Non-linear Mech. 15, 147-154, 1980.

[11] S.K. Gupta, R. L. Dharmani, Creep transition in thick walled cylinder under internal pressure, Z. Angew. Math. Mech. 59, 517-521, 1979.
[12] N.I. Muskhelishvili, Some basic problems of the mathematical theory of elasticity, Noordhoff, Groningen, 1953.

[13]N. Khomasuridze, Thermoelastic equilibrium of bodies in generalized cylindrical coordinates, Georgian Math. J. 5, 521-544, 1998.

[14] N. Khomasuridze, N. Zirakashvili, Strain control of cracked elastic bodies by means of boundary condition variation, In: Proceedings of International Conference "Architecture and Construction Contemporary Problems', 30 September-3 October, Yerevan, Jermuk, 158-163, 2010 (in Russian).

[15] N. Zirakashvili, Application of the boundary element method to the solution of the problem of distribution of stresses in an elastic body with a circular hole whose interior surface contains radial cracks, Proc.

A. Razmadze Math. Inst. 141, 139-147, 2006.

[16] R. Tang, Y. Wang, On the problem of crack system with an elliptic hole, Acta Mech. Sin. 2, 1, 47-53, 1986.

[17] N. Zirakashvili, Some boundary value problems of elasticity for semi-ellipses, Proc. I. Vekua Inst. Appl. Math. 52, 49-55, 2002.

[18] Y. Shestopalov, N. Kotik, Approximate decomposition for the solution of boundary value problems for elliptic systems arising in mathematical models of layered structures, In: Progress in Electromagnetic Research Symposium, Cambridge, MA, 26-29 March, 514-518, 2006.

[19] N. Zirakashvili, The numerical solution of boundary value problems for an elastic body with an elliptic hole and linear cracks, J. Eng. Math. 65, 2, 111-123, 2009.

[20]N. Zirakashvili, On the numerical solution of some two-dimensional boundary-contact delocalization problems. Meccanica, 48, 7, 1791-1804, 2013.

[21]N. Zirakashvili, Boundary Value Problems of Elasticity for Semi-ellipse with Non- homogeneous Boundary Conditions at the Segment Between Focuses, Bulletin of TICMI, 21, 2, 95-116, 2017.

[22] N. Zirakashvili, Study of deflected mode of ellipse and ellipse weakened with crack, ZAMM, 97, 8, 932$945,2017$.

[23] N. Zirakashvili, Analytical solutions of some internal boundary value problems of elasticity for domains with hyperbolic boundaries, Mathematics and Mechanics of Solids, 24, 6, 1726-1748, 2019.

[24]N. Zirakashvili, Analytical solutions of boundaryvalue problems of elasticity for confocal elliptic ring and its parts, Journal of the Brazilian Society of 
Mechanical Sciences and Engineering, 40, 8, article: 398 1-19, 2018.

[25] N. Zirakashvili, Study of stress-strain state of elastic body with hyperbolic notch, Z. Angew. Math. Phys. 70:87, 1-19, 2019.

[26] N. Zirakashvili, Analytical solution of interior boundary value problems of elasticity for the domain bounded by the parabola, Bulletin of TICMI, 20, 1, 3-24, 2016.

[27] N. Zirakashvili, Exact solution of some exterior boundary value problems of elasticity in parabolic coordinates, Mathematics and Mechanics of Solids, 23, 6, 929-943, 2018.

[28] G.B. Jeffery, Planes tress and planes train in bipolar coordinates, Trans. R. Soc. Lon. 221, 265-293, 1921.

[29] Ya.S. Ufland, Bipolar Coordinates in Elasticity, Gostehteoretizdat, Moscow-Leningrad, 1950 (in Russian).

[30]N. Khomasuridze, Solution of some elasticity boundary value problems in bipolar coordinates, Acta Mechanica, 189, 207-224, 2007.

[31] M. Filonenko-Borodich, Theory of Elasticity, Gos. Izd. Phiz. -Mat. Lit. Moscow, 1959 (in Russian).

[32]N. Khomasuridze, Representation of solutions of some boundary value problems of elasticity by a sum of the solutions of other boundary value problems, Georgian Mathematical Journal, 10, 2, 257-270, 2005.

[33] W. Nowacki, Elasicity Theory, Mir, Moscow, 1975 (in Russian).

[34] V.I. Feodoseev, Strength of materials. Nauka, Moscow, 1972 (in Russian).

[35] G.S. Shpiro, A.V. Darkov, Strength of materials. Vishaia shkola, Moscow, 1989 (in Russian).

\section{Creative Commons Attribution License 4.0 (Attribution 4.0 International, CC BY 4.0)}

This article is published under the terms of the Creative Commons Attribution License 4.0

https://creativecommons.org/licenses/by/4.0/deed.en_US 